\title{
PSEUDODIFFERENTIAL PARABOLIC EQUATIONS; TWO EXAMPLES
}

\author{
Tomasz Dlotko - Maria B. Kania - Chunyou Sun
}

\begin{abstract}
The paper is devoted to local and global solvability and existence of a global attractor for an exemplary 'parabolic' problem containing fractional powers of the minus Laplace operator. We want to compare, which properties of the similar semilinear heat equation are preserved when we replace the pure minus Laplace operator by a fractional power of that operator. Several useful technical tools and estimates are collected in that paper.
\end{abstract}

\section{Introduction}

Our aim is to study a pseudodifferential equation having fractional power of the minus Laplacian as a main part operator. Such equation describes the process of anomalous diffusion that has been extensively studied in recent years (see e.g. [4], [5], [15], [19], [24]) both in mathematical and in physical context. The fractal diffusion has been used to describe various phenomena in statistical mechanics, hydrodynamics, acoustics and biology. It also appears in nonlinear models of interfacial growth which involve hopping and trapping effects.

Our task in the present paper is to provide mathematical analysis of equation (1.1) including existence, uniqueness and asymptotic behavior of solutions both for the Dirichlet problem in bounded domain and for the Cauchy problem in $\mathbb{R}^{N}$. More precisely, we will concentrate on the two 'difficult cases'; Cauchy's

2010 Mathematics Subject Classification. 35S10, 35S15, 35B41, 35K58.

Key words and phrases. Fractal parabolic equation, fractional powers of sectorial operator, monotone operator, global solution, global attractor. 
problem in the whole of $\mathbb{R}^{N}$ with a supercritical nonlinearity, and Dirichlet's problem in bounded domain when the nonlinearity is of critical growth. In Section 2 we discuss local and global solvability of the considered problems. Section 3 contains a number of useful tools and estimates suitable in further studies. We recall there, from [16], [13], some estimates of the Kato-Beurling-Deny type for the case of bounded domain and under the Balakrishnan definition of the fractional powers of operators (see [25]). Corresponding estimates in the case of $\mathbb{R}^{N}$, called Strook-Varopoulos inequalities, obtained under the Definition 3.6 of the fractional powers of $(-\Delta)$ on $\mathbb{R}^{N}$, can be found in the review article [24]. We present also, in Section 3, variants of the Moser-Alikakos type estimates (see [1]), both in bounded domain and in $\mathbb{R}^{N}$. Section 4 is devoted to existence of the global attractor, in $L^{2}\left(\mathbb{R}^{N}\right)$, for the case of Cauchy's problem. We are using there the 'tail estimate technique' (see [33]). Technical tools and estimates collected in Section 3 are strongly used throughout the whole text of the paper.

We refer to [7], [4], [5], [18], [19], [24] for more complete introduction to the theory of equations with fractional powers of the Laplacian and for description of the role of anomalous diffusion in physical phenomena.

EXAMPLE 1.1. As a first example consider the fractal dissipative equation:

$$
\begin{cases}u_{t}+(-\Delta)^{\alpha} u+f(u)+\lambda u=g(x), & t>0, x \in \mathbb{R}^{N}, \\ u(0, x)=u_{0}(x), & x \in \mathbb{R}^{N},\end{cases}
$$

with $\lambda>0, \alpha \in(0,1)$ and $N \geq 3$; where $g \in L^{2}\left(\mathbb{R}^{N}\right)$ and the nonlinearity $f \in C^{1}(\mathbb{R})$ satisfies the following conditions:

$$
\begin{aligned}
& \exists 0<k_{1}<\lambda \quad \forall s \in \mathbb{R} \\
& \quad c_{1}|s|^{p+1}-k_{1}|s|^{2} \leq f(s) s \leq c_{2}|s|^{p+1}+k_{2}|s|^{2}, \\
& \exists l<\lambda \quad \forall s \in \mathbb{R} \quad f^{\prime}(s) \geq-l .
\end{aligned}
$$

The exponent $p \geq 1$ is arbitrary (compare [34] for similar supercritical problems). It is easy to see that with the above assumptions the function $f$ will be decomposed as:

$$
f(s)=f_{1}(s)+f_{2}(s),
$$

where $f_{1}(s)=f(s)+\left(k_{1}+\lambda\right) s$ and $f_{2}=-\left(k_{1}+\lambda\right) s$. Evidently $f_{1}$ defines a monotone operator since:

$$
f_{1}^{\prime}(s) \geq-l+\lambda+k_{1}>0 \quad \text { for all } s \in \mathbb{R}
$$

thanks to the assumption (1.3). Moreover, the function $f_{2}$ is linear, hence globally Lipschitz. 
EXAMPLE 1.2. As a second example consider the fractal dissipative equation:

$$
\begin{cases}u_{t}+(-\Delta)^{\alpha} u+f(u)=g(x), & t>0, x \in \Omega, \\ u(0, x)=u_{0}(x), & x \in \Omega,\left.u\right|_{\partial \Omega}=0\end{cases}
$$

with $\alpha \in(0,1)$ and $N \geq 3$; where $g \in H^{-\alpha}(\Omega)$, and $\Omega \subset \mathbb{R}^{N}$ is a bounded domain with smooth (at least of class $C^{2}$ ) boundary. Moreover, we assume that

$$
\liminf _{|s| \rightarrow \infty} \frac{f(s)}{s}>-\lambda_{1}
$$

and

$$
\lim _{|s| \rightarrow \infty} \frac{\left|f^{\prime}(s)\right|}{|s|^{2 \alpha p /(N-\alpha p)}}=0,
$$

where $\lambda_{1}$ is the first eigenvalue of $(-\Delta)^{\alpha}$ in $\Omega$ with Dirichlet boundary condition.

\section{The existence and uniqueness of solutions}

2.1. Cauchy's problem in $\mathbb{R}^{N}$. We start with considering the first example (1.1). We will prove local solvability of that problem following the approach of [9]. The solution will be obtained in the phase space $H^{\alpha}\left(\mathbb{R}^{N}\right) \cap L^{p+1}\left(\mathbb{R}^{N}\right)$. The 'abstract' operator $A$ on $H^{\alpha}\left(\mathbb{R}^{N}\right) \cap L^{p+1}\left(\mathbb{R}^{N}\right), \alpha \in(0,1)$, will correspond to differential operator $(-\Delta)^{\alpha} u+f_{1}(u)+\lambda u$.

An abstract problem with monotone operator

$$
\begin{gathered}
\frac{d u(t)}{d t}+A(u(t))+B(u(t))=0, \quad t>0, \\
u(0)=u_{0} \in H
\end{gathered}
$$

was considered in [9]. We formulate:

Definition 2.1. A function $u \in C([0, T] ; H)$ is a strong solution to $(2.1)$ if $u$ is absolutely continuous in any compact subinterval of $(0, T), u(t) \in \mathcal{D}\left(A_{H}\right)$ for almost all $t \in(0, T)$ and

$$
\frac{d u}{d t}(t)+A(u(t))+B(u(t))=0 \quad \text { for a.a. } t \in(0, T) .
$$

A function $u \in C([0, T] ; H)$ is called a weak solution to $(2.1)$ if there is a sequence $\left\{u_{n}\right\}$ of strong solutions convergent to $u$ in $C([0, T] ; H)$.

It was assumed there that:

(H1) (i) $H$ is a Hilbert space and $V$ is a reflexive Banach space such that $V \subset H \subset V^{*}$, with continuous inclusions. Moreover, $V$ is dense in $H$.

(ii) $A$ is a nonlinear, monotone, coercive and hemicontinuous operator such that $A: V \rightarrow V^{*}$ (defined on the whole of $V$ ). 
(iii) The operator $B(u)$ (where $u \in H$ ) is globally Lipschitz from $H$ into $H$.

REMARK 2.2. In [9] to obtain density of the domain of $A$ in $H$ the condition (H2) was also assumed. In the present paper such a density is evident, so that we call (H2) for completeness of the presentation.

(H2) There are constants $\omega_{1}, \omega_{2}>0, c_{1} \in \mathbb{R}$ and $P \geq 2$ such that for all $v \in V$ the following two conditions hold:

$$
\langle A v, v\rangle_{V^{*}, V} \geq \omega_{1}\|v\|_{V}^{P}+c_{1}, \quad\|A v\|_{V^{*}} \leq \omega_{2}\left(1+\|v\|_{V}^{P-1}\right) .
$$

Recall, [20], that the operator $A: V \rightarrow V^{*}$ is called:

- monotone, if for arbitrary $u, v \in V$

$$
\langle A u-A v, u-v\rangle_{V^{*}, V} \geq 0 .
$$

- coercive, if

$$
\lim _{\|v\|_{V} \rightarrow+\infty} \frac{\langle A v, v\rangle_{V^{*}, V}}{\|v\|_{V}}=+\infty .
$$

- hemicontinuous, if for arbitrary fixed $u, v, h \in V$ the real function

$$
s \rightarrow\langle A(u+s v), h\rangle_{V^{*}, V} \text { is continuous on }[0,1] .
$$

With the above assumption (H1) existence of a solution was shown in [9] (see also [6]). More precisely, we quote:

Proposition 2.3. Denote $D\left(A_{H}\right):=\{v \in V: A(v) \in H\}$. Then, under the assumptions $(\mathrm{H} 1)(\mathrm{i})$, (ii) and $(\mathrm{H} 2)$ the set $D\left(A_{H}\right)$ is dense in $H$. Moreover, under the sole hypotheses (H1), the equation (2.1) defines a semigroup of nonlinear operators $S(t): \mathrm{cl}_{H}\left(D\left(A_{H}\right)\right) \rightarrow \mathrm{cl}_{H}\left(D\left(A_{H}\right)\right), t \geq 0$, where for each $u_{0} \in \operatorname{cl}_{H}\left(D\left(A_{H}\right)\right)$

$$
t \rightarrow S(t) u_{0}
$$

is the global weak solution of (2.1) starting at $u_{0}$. This semigroup is such that

$$
\mathbb{R}^{+} \times \operatorname{cl}_{H}\left(D\left(A_{H}\right)\right) \ni\left(t, u_{0}\right) \rightarrow S(t) u_{0} \in \operatorname{cl}_{H}\left(D\left(A_{H}\right)\right)
$$

is a continuous map. Moreover, for $u_{0} \in D\left(A_{H}\right), u(\cdot)=S(\cdot) u_{0}$ is a Lipschitz continuous strong solution of (2.1).

We are now ready to apply the quoted above result to our present problem (1.1). Setting:

$$
\begin{aligned}
H & =L^{2}\left(\mathbb{R}^{N}\right), \quad V=H^{\alpha}\left(\mathbb{R}^{N}\right) \cap L^{p+1}\left(\mathbb{R}^{N}\right), \\
A(u) & =(-\Delta)^{\alpha} u+f_{1}(u)+\lambda u, \\
B(u) & =f_{2}(u)-g(\cdot),
\end{aligned}
$$

validity of the condition (H1) in that case will be checked next. 
First note, that the space $V$ is reflexive thanks to the equality $\left(H^{\alpha}\left(\mathbb{R}^{N}\right) \cap\right.$ $\left.L^{p+1}\left(\mathbb{R}^{N}\right)\right)^{* *}=H^{\alpha}\left(\mathbb{R}^{N}\right) \cap L^{p+1}\left(\mathbb{R}^{N}\right)$ (see [20], Theorem 5.13). The operator $A$ is connected with the duality form

$$
\langle A(u), v\rangle_{V^{*}, V}=\int_{\mathbb{R}^{N}}(-\Delta)^{\alpha / 2} u(-\Delta)^{\alpha / 2} v d x+\int_{\mathbb{R}^{N}}\left(f_{1}(u)+\lambda u\right) v d x, \quad u, v \in V .
$$

Since $f_{1}$ defines a monotone operator it is evident that the operator $A$ is monotone itself. Moreover, the operator $A$ is coercive and hemicontinuous. Indeed, thanks to (1.2) and the Young inequality, we have

$$
\begin{aligned}
\langle A(v), v\rangle_{V^{*}, V} & \geq c_{\lambda}\|v\|_{H^{\alpha}\left(\mathbb{R}^{N}\right)}^{2}+\int_{\mathbb{R}^{N}} f_{1}(v) v d x \\
& \geq c_{\lambda}\|v\|_{H^{\alpha}\left(\mathbb{R}^{N}\right)}^{2}+c_{1}\|v\|_{L^{p+1}\left(\mathbb{R}^{N}\right)}^{p+1} \\
& \geq c\left(\|v\|_{H^{\alpha}\left(\mathbb{R}^{N}\right)}^{2}+\|v\|_{L^{p+1}\left(\mathbb{R}^{N}\right)}^{2}-1\right) \\
& \geq c\|v\|_{V}^{2}-c=\frac{c\|v\|_{V}^{2}-c}{\|v\|_{V}}\|v\|_{V},
\end{aligned}
$$

where $c_{\lambda}$ is a constant appearing in the equivalent norm:

$$
c_{\lambda}\|u\|_{H^{\alpha}\left(\mathbb{R}^{N}\right)}^{2} \leq\left\|(-\Delta)^{\alpha / 2} u\right\|_{L 2\left(\mathbb{R}^{N}\right)}^{2}+\lambda\|u\|_{L^{2}\left(\mathbb{R}^{n}\right)}^{2} \quad \text { for all } u \in H^{\alpha}\left(\mathbb{R}^{N}\right) .
$$

Recall next that the norm in the dual space $V^{*}=H^{-\alpha}\left(\mathbb{R}^{N}\right)+L^{(p+1) / p}\left(\mathbb{R}^{N}\right)$ is given by the formula:

$$
\|z\|_{V^{*}}=\inf _{\substack{x \in H^{-\alpha}\left(\mathbb{R}^{N}\right), y \in L^{(p+1) / p}\left(\mathbb{R}^{N}\right) \\ z=x+y}} \max \left(\|x\|_{H^{-\alpha}\left(\mathbb{R}^{N}\right)},\|y\|_{L^{(p+1) / p}\left(\mathbb{R}^{N}\right)}\right) .
$$

Note that in our case we have the characterizations: $D\left((-\Delta)^{\alpha / 2}\right)=H^{\alpha}\left(\mathbb{R}^{N}\right)$, and under the assumption (1.2) the domain of the Nemitskii operator connected to $f_{1}$ contains $L^{p+1}\left(\mathbb{R}^{N}\right)$. Consequently, the domain of the whole operator $A$ contains $H^{\alpha}\left(\mathbb{R}^{N}\right) \cap L^{p+1}\left(\mathbb{R}^{N}\right)$ and is dense in $H=L^{2}\left(\mathbb{R}^{N}\right)$.

Corollary 2.4. As a consequence of the Proposition 2.3 we conclude existence of a solution to (1.1). More precisely, for $u_{0} \in D\left(A_{H}\right)=\left\{v \in H^{\alpha}\left(\mathbb{R}^{N}\right) \cap\right.$ $\left.L^{p+1}\left(\mathbb{R}^{N}\right) ; A(v) \in L^{2}\left(\mathbb{R}^{N}\right)\right\}$, the corresponding to $u_{0}$ weak solution $u(t)$ satisfies:

$$
u(t) \in C\left((0, T) ; L^{2}\left(\mathbb{R}^{N}\right)\right) \quad \text { for all } T>0 .
$$

Moreover, since $u_{0} \in D\left(A_{H}\right)$ then $u(t)$ is in fact a strong solution to (1.1); it is absolutely continuous in any compact subinterval of $(0, T), u(t) \in D\left(A_{H}\right)$ and (1.1) is fulfilled in $L^{2}\left(\mathbb{R}^{N}\right)$ for almost all $t \in(0, T)$.

REMARK 2.5. The assumption of the global Lipschitz continuity of the function $f_{2}$ will be weakened if one uses the results of [11] instead of [9]. 
2.2. More regular solutions in $\mathbb{R}^{N}$. Analogously as in the theory of second order parabolic equations, if we consider the problem (1.1) on a smaller phase space, then it will not be critical anymore and can be treated within the classical approach of [22]. Consider thus the Cauchy problem (1.1) with nonlinearity $f: \mathbb{R} \rightarrow \mathbb{R}$ locally Lipschitz continuous, $g \in L^{\infty}\left(\mathbb{R}^{N}\right) \cap L^{2}\left(\mathbb{R}^{N}\right)$ in the phase space $H_{p+1}^{\beta}\left(\mathbb{R}^{N}\right)$, with $2 \alpha>\beta>N /(p+1)$. Because of the last restriction on $\beta$ and $p$ it is seen that $H_{p+1}^{\beta}\left(\mathbb{R}^{N}\right) \subset L^{\infty}\left(\mathbb{R}^{N}\right)$.

In the base space $X=L^{p+1}\left(\mathbb{R}^{N}\right)$ consider the $X^{\beta /(2 \alpha)}$-solution to (1.1) in a sense of [22]. Sectoriality of the realization of $(-\Delta)^{\alpha}$ in that case follows from the known sectoriality of $(-\Delta)$ alone (see [22, p. 33]) and Proposition 2.11. To get the $X^{\beta /(2 \alpha)}$-solution it is thus enough to check that the 'nonlinear term'

$$
F(v)=-f(v)-\lambda v+g(\cdot),
$$

is Lipschitz continuous on bounded sets as a map from $X^{\beta /(2 \alpha)}$ to $X$. But the last property is evident thanks to the embedding $X^{\beta /(2 \alpha)} \subset L^{\infty}\left(\mathbb{R}^{N}\right)$ and the local Lipschitz continuity of the real function $f$. The solution obtained vary continuously in $X^{\beta /(2 \alpha)}$, so also in $L^{\infty}\left(\mathbb{R}^{N}\right)$. As usual, to show global in time extendibility of the $X^{\beta /(2 \alpha)}$-local solution, we need first to get a priori estimate of it in an auxiliary Banach space. In our case we choose $Y=L^{\infty}\left(\mathbb{R}^{N}\right) \cap L^{p+1}\left(\mathbb{R}^{N}\right)$. Note that the a priori estimate in that space is a direct consequence of the estimate (3.15) and the Moser-Alikakos estimates (compare [1], [12, p. 213]) presented in Subsection $3.2((3.13))$. Next, we show that the subordination condition for the nonlinear term holds. Note that as a direct consequence of the assumption (1.2) we get

$$
\exists c_{3}>0 \quad \forall s \in \mathbb{R} \quad|f(s)| \leq c_{3}\left(|s|^{p}+|s|\right) .
$$

Then, thanks to (2.3) and the Young inequality, we have

$$
\begin{aligned}
\|F(u)\|_{L^{p+1}\left(\mathbb{R}^{N}\right)} & \leq C\|u\|_{L^{\infty}\left(\mathbb{R}^{N}\right)}^{p-1}\|u\|_{L^{p+1}\left(\mathbb{R}^{N}\right)}+(C+\lambda)\|u\|_{L^{p+1}\left(\mathbb{R}^{N}\right)}+\|g\|_{L^{p+1}} \\
& \leq C\left(\|u\|_{L^{\infty}\left(\mathbb{R}^{N}\right) \cap L^{p+1}\left(\mathbb{R}^{N}\right)}^{p}+\|u\|_{L^{\infty}\left(\mathbb{R}^{N}\right) \cap L^{p+1}\left(\mathbb{R}^{N}\right)}+1\right) \\
& =c\left(\|u\|_{Y}\right)
\end{aligned}
$$

which is a simple form of the subordination condition as in [12]. We have thus proved existence of a smooth solution to (1.1):

Proposition 2.6. Let the function $f: \mathbb{R} \rightarrow \mathbb{R}$ be locally Lipschitz continuous, $g \in L^{\infty}\left(\mathbb{R}^{N}\right) \cap L^{2}\left(\mathbb{R}^{N}\right)$, then there exists a unique solution $u \in H_{p+1}^{\beta}\left(\mathbb{R}^{N}\right), 2 \alpha>$ $\beta>N /(p+1)$, of (1.1), such that:
(a) $u \in C\left([0, T] ; H_{p+1}^{\beta}\left(\mathbb{R}^{N}\right)\right)$,
(b) $u \in C^{1}\left((0, T) ; H_{p+1}^{2 \alpha}\left(\mathbb{R}^{N}\right)\right), u_{t} \in H_{p+1}^{2 \alpha}\left(\mathbb{R}^{N}\right)$ for all $t \in(0, T)$,
(c) the equation is satisfied in $L^{p+1}\left(\mathbb{R}^{N}\right)$ for all $t \in(0, T)$,
(d) the solution vary continuously in $L^{\infty}\left(\mathbb{R}^{N}\right)$. 
2.3. The problem in bounded domain. In this section we consider the problem (1.4) in bounded domain $\Omega \subset \mathbb{R}^{N}$ in case when the nonlinearity has critical growth. To handle such a problem in $H_{p}^{-\alpha}(\Omega)$ (with nonlinearity growing with 'critical' exponent $(N+\alpha p) /(N-\alpha p))$ we need to use the notion of an $\varepsilon$ regular solution as introduced in [3]. We will recall next the basic definitions and results concerning $\varepsilon$-regular solutions (see [3] for more details). We start with some terminology.

Let $X$ be a Banach space and $A: D(A) \subset X \rightarrow X$ be a sectorial operator with $\operatorname{Re} \sigma(A)>0$. Then, $-A$ generates an analytic semigroup denoted by $\left\{e^{-A t}\right.$ : $t \geq 0\}$. Let $X^{\beta}:=D\left(A^{\beta}\right), \beta \geq 0$, endowed with the graph norm, be the fractional power spaces associated with $A$. Consider the semilinear differential equation

$$
\begin{gathered}
u_{t}+A u=F(u), \quad t>0, \\
u(0)=u_{0} \in X^{1},
\end{gathered}
$$

where $F: D(F) \subset X^{1} \rightarrow X^{\beta}$ for some $\beta>0$.

Definition $2.7([3])$. For $\varepsilon>0$, a function $u:[0, \tau) \rightarrow X^{1}$ is called an $\varepsilon$-regular solution for $(2.4)$ if $u \in C\left([0, \tau) ; X^{1}\right) \cap C\left((0, \tau) ; X^{1+\varepsilon}\right)$ and

$$
u(t)=e^{-A t} u_{0}+\int_{0}^{t} e^{-A(t-s)} F(u(s)) d s, \quad t \in[0, \tau) .
$$

Definition 2.8 ([3], [8]). The map $F$ is called a critical $\varepsilon$-regular map relative to the pair $\left(X^{1}, X\right)$, if there are positive constants $c, \eta, C_{\eta}, q>1$ and $\varepsilon \in(0,1 / q)$ such that, for each $v, w \in X^{1+\varepsilon}$,

$$
\begin{gathered}
\|F(v)-F(w)\|_{X^{q \varepsilon}} \leq c\|v-w\|_{X^{1+\varepsilon}}\left(C_{\eta}+\eta\|v\|_{X^{1+\varepsilon}}^{q-1}+\eta\|w\|_{X^{1+\varepsilon}}^{q-1}\right), \\
\|F(v)\|_{X^{q \varepsilon}} \leq c\left(C_{\eta}+\eta\|v\|_{X^{1+\varepsilon}}^{q}\right) \quad \text { for } v \in X^{1+\varepsilon}
\end{gathered}
$$

In addition, if for each $\eta>0$ there is $C_{\eta}>0$ such that (2.6) holds with $c, q$ and $\varepsilon$ independent of $\eta$, then $F$ is called an almost critical $\varepsilon$-regular map relative to the pair $\left(X^{1}, X\right)$.

After this introduction we can state a variant of Theorem 2.1 in [8]:

Proposition 2.9. Let $F$ be a critical $\varepsilon$-regular map. Fixing $v_{0} \in X^{1}$, there are $r>0$ and $\tau_{0}>0$ such that for each $u_{0} \in B_{X^{1}}\left(v_{0}, r\right)$ there exists a unique $\varepsilon$-regular solution $u$ of $(2.4)$ defined in $\left[0, \tau_{0}\right]$. In addition,

(a) $t^{\xi}\left\|u\left(t, u_{0}\right)\right\|_{X^{1+\xi}} \rightarrow 0$ as $t \rightarrow 0^{+}, 0<\xi<q \varepsilon$,

(b) $t^{\xi}\left\|u\left(t, u_{1}\right)-u\left(t, u_{2}\right)\right\|_{X^{1+\xi}} \leq C^{\prime}\left\|u_{1}-u_{2}\right\|_{X^{1}}$ for $t \in\left[0, \tau_{0}\right], 0 \leq \xi \leq \xi_{0}<$ $q \varepsilon, u_{1}, u_{2} \in B_{X^{1}}\left(v_{0}, r\right)$,

(c) $u\left(t, u_{0}\right) \in C\left(\left(0, \tau_{0}\right] ; X^{1+q \varepsilon}\right) \cap C^{1}\left(\left(0, \tau_{0}\right] ; X^{1+\xi}\right)$ for $0 \leq \xi<q \varepsilon$; in particular the solution $u\left(t, u_{0}\right)$ satisfies $(2.4)$ for each $t \in\left(0, \tau_{0}\right]$. 
If $F$ is an almost critical $\varepsilon$-regular map, then all the above holds for arbitrarily large $r>0$. In this case, if the solution $u\left(t, u_{0}\right)$ is bounded in $X^{1}$ in its maximal interval of existence, it must exist for all $t \geq 0$.

Usually the above mentioned result is used in case when the sectorial operator $A$ is given by the differential operator $-\Delta$ or a more general elliptic differential operator (subjected to a suitable boundary condition). But we will use it here in the case when $A$ will be a proper $\alpha$ fractional power, $\alpha \in(0,1)$, of an elliptic operator (so, it is a pseudodifferential operator).

It is well known that fractional powers of sectorial positive operators are, for $\alpha \in(0,1)$, sectorial and positive. To verify the last claim it is convenient to use a notion, due to $\mathrm{H}$. Komatsu, of an operator of the type $(\omega, M(\theta))$ with $\omega<\pi / 2$ in a Banach space $X$.

Definition 2.10. We say that $A$ is of type $(\omega, M(\theta)), 0 \leq \omega<\pi$, if the domain $D(A)$ is dense in $X$, the resolvent set of $-A$ contains the sector $|\arg \lambda|<$ $\pi-\omega$ and the condition $\left\|\lambda(\lambda+A)^{-1}\right\| \leq M(\theta)$ holds on each ray $\lambda=\operatorname{re}^{i \theta}$, $r \in(0,+\infty),|\theta|<\pi-\omega$.

One may easily verify that $A$ is of the type $(\omega, M(\theta))$ if and only if $A$ is a sectorial positive operator in the sense of [22]. A theorem by T. Kato (see [25, p. 320]) ensures that:

Proposition 2.11. If $A$ is of type $(\omega, M(\theta))$ and if $0<\alpha<\pi / \omega$, then $A^{\alpha}$ is of type $\left(\alpha \omega, M_{\alpha}(\theta)\right)$ with certain positive constant $M_{\alpha}(\theta)$. Furthermore, the resolvent of $A^{\alpha}$ is analytic in $\alpha$ and $\lambda$ in the domain $0<\alpha<\pi / \omega,|\arg \lambda|<$ $\pi-\alpha \omega$.

As a consequence, any proper fractional power $A^{\alpha}, \alpha \in(0,1)$, of a sectorial positive operator $A$ will be sectorial and positive itself. Furthermore,

Observation 2.12. If $A$ is of type $(\omega, M(\theta))$ with $\omega<\pi / 2$, then the sum $A+A^{\beta}$ is a sectorial operator for any $\beta \in(0,1)$.

The above observation follows directly from [22, Theorem 1.4.4] and [22, Example 6, p. 19] since

$$
\forall \varepsilon>0 \quad \forall \beta \in(0,1) \quad \forall x \in D(A) \quad\left\|A^{\beta} x\right\|_{X} \leq \varepsilon\|A x\|_{X}+C^{\prime} \varepsilon^{-\beta /(1-\beta)}\|x\|_{X} .
$$

Once we take care for checking that the main operator $(-\Delta)^{\alpha}$ (Dirichlet boundary condition) in (1.4) is sectorial, we need to verify that the nonlinear term $f$ generates a critical $\varepsilon$-regular map. We set $X=H_{p}^{-\alpha}(\Omega), A=(-\Delta)^{\alpha}$, where $\alpha \in(0,1)$ is fixed, so that $X^{1}=H_{p,\{D\}}^{\alpha}(\Omega)$ is the associated space of Bessel potentials (see [32, Section 2.3.1] for more information concerning that spaces); the lower index $D$ corresponds to the homogeneous Dirichlet condition. 
In what follows, we apply these abstract results to obtain local well posedness of (1.4) in $H_{p,\{D\}}^{\alpha}(\Omega)$. Since $\Omega$ is smooth we have that the domains of fractional power scale associated to $A$ coincide with the complex interpolation scale (see [2], [32, Section 4.9.2]). In particular ([32, Section 4.3.3]), for $0<\theta<1$,

$$
\left[L^{p}(\Omega), H_{p,\{D\}}^{m}(\Omega)\right]_{\theta}=H_{p,\{D\}}^{\theta m}(\Omega)
$$

whenever $\theta m \neq 1 / p$ (here $[\cdot, \cdot]_{\theta}$ denotes the complex interpolation space of order $\theta)$.

Now, we check that the condition (2.6) of Definition 2.8 is satisfied. To formulate the result we need to use the assumption (1.6):

$$
\lim _{|s| \rightarrow \infty} \frac{\left|f^{\prime}(s)\right|}{|s|^{2 \alpha p /(N-\alpha p)}}=0
$$

A nonlinearity satisfying such condition is called almost critical. The value $q=$ $(N+\alpha p) /(N-\alpha p)$ is called a critical exponent here.

Note that if $f \in C^{1}(\mathbb{R}, \mathbb{R})$ satisfies (1.6), then for each $\eta>0$ there exists $C_{\eta}>0$ such that

$$
\left|f\left(s_{1}\right)-f\left(s_{2}\right)\right| \leq\left|s_{1}-s_{2}\right|\left(C_{\eta}+\eta\left|s_{1}\right|^{q-1}+\eta\left|s_{2}\right|^{q-1}\right), \quad s_{1}, s_{2} \in \mathbb{R},
$$

and also, for each $\eta>0$ there exists $\widetilde{C}_{\eta}>0$ such that

$$
|f(s)| \leq \widetilde{C}_{\eta}+\eta|s|^{q} .
$$

Let $F$ be the Nemytskii operator corresponding to $-f(\cdot)+g$, then the following estimates are satisfied.

Lemma 2.13. Let $N \geq 3, \alpha p<N, q:=(N+\alpha p) /(N-\alpha p)$ and $f: \mathbb{R} \rightarrow \mathbb{R}$ be a continuously differentiable function which satisfies (1.6) and $g \in L^{r / q}(\Omega)$. For $\nu>0$ and $\varepsilon \in[0, \alpha /(2 q))$, there is a constant $c>0$ and, for each $\eta>0$, a constant $\widetilde{C}_{\eta}>0$ such that

$$
\left\|F\left(w_{1}\right)-F\left(w_{2}\right)\right\|_{X^{q \varepsilon}} \leq c\left\|w_{1}-w_{2}\right\|_{X^{1+\varepsilon}}\left(\widetilde{C}_{\eta}+\eta\left\|w_{1}\right\|_{X^{1+\varepsilon}}^{q-1}+\eta\left\|w_{2}\right\|_{X^{1+\varepsilon}}^{q-1}\right),
$$

for $w_{1}, w_{2} \in X^{1+\varepsilon}$. Also, the second estimate in (2.6) is satisfied by $F$.

Proof. It follows from Sobolev embedding theorem that

$$
H_{p,\{D\}}^{\alpha+2 \varepsilon}(\Omega)=X^{1+\varepsilon} \subset L^{r}(\Omega), \quad L^{r / q}(\Omega) \subset X^{q \varepsilon}(\Omega)=H_{p,\{D\}}^{-\alpha+2 q \varepsilon}(\Omega) .
$$

for $r:=N p /(N-p(2 \varepsilon+\alpha))$, Next, from (2.7) and the Hölder inequality,

$$
\begin{aligned}
\left\|F\left(w_{1}\right)-F\left(w_{2}\right)\right\|_{X^{q \varepsilon}} & \leq c\left\|f\left(w_{1}\right)-f\left(w_{2}\right)\right\|_{L^{r / q}(\Omega)} \\
& \leq c\left\|w_{1}-w_{2}\right\|_{L^{r}(\Omega)}\left(\widetilde{C}_{\eta}+\eta\left\|w_{1}\right\|_{L^{r}(\Omega)}^{q-1}+\eta\left\|w_{2}\right\|_{L^{r}(\Omega)}^{q-1}\right) \\
& \leq c\left\|w_{1}-w_{2}\right\|_{X^{1+\varepsilon}}\left(\widetilde{C}_{\eta}+\eta\left\|w_{1}\right\|_{X^{1+\varepsilon}}^{q-1}+\eta\left\|w_{2}\right\|_{X^{1+\varepsilon}}^{q-1}\right) .
\end{aligned}
$$


The proof of the second estimate in (2.6) is similar; thanks to (2.8) we have

$$
\begin{aligned}
\|F(w)\|_{X^{q \varepsilon}} & \leq c\|f(w)\|_{L^{r / q}(\Omega)}+\|g\|_{L^{r / q}(\Omega)} \\
& \leq c\left[\int_{\Omega}\left[\widetilde{C}_{\eta}+\eta|w|^{q}\right]^{r / q} d x\right]^{q / r}+\|g\|_{L^{r / q}(\Omega)} \\
& \leq c\left(\widetilde{C}_{\eta}+\eta\|w\|_{L^{r}(\Omega)}^{q}\right)+\|g\|_{L^{r / q}(\Omega)} .
\end{aligned}
$$

The proof of the lemma is now complete.

Conclusions. We have thus verified all the conditions necessary to apply the abstract Proposition 2.9 to our present problem (1.4). Consequently, existence of a local in time $\varepsilon$-regular solution $u$ to (1.4) follows having all the properties as specified in Definition 2.7 and Proposition 2.9. In particular $u \in C\left([0, \tau) ; H_{p,\{D\}}^{\alpha}(\Omega)\right) \cap C\left((0, \tau) ; H_{p,\{D\}}^{\alpha+2 \varepsilon}(\Omega)\right)$ and the equation is fulfilled in $H_{p,\{D\}}^{-\alpha}(\Omega)$.

Let us restrict, for simplicity, further studies of the problem (1.4) to the Hilbert case $p=2$. As a consequence of the condition (1.6) the local $\varepsilon$-regular solution will be extended globally in time. The last property follows from existence of the Lyapunov function obtained through multiplication of (1.4) by $u_{t}$. As a result of that multiplication we obtain

$$
\frac{d}{d t}\left(\frac{1}{2} \int_{\Omega}\left[(-\Delta)^{\alpha / 2} u\right]^{2} d x+\int_{\Omega} F(u) d x-\int_{\Omega} g(x) u d x\right)+\int_{\Omega} u_{t}^{2} d x=0
$$

where $F(s)=\int_{0}^{s} f(z) d z$ is a primitive of $f$. Consequently, the expression

$$
\mathcal{L}(u(t))=\frac{1}{2} \int_{\Omega}\left[(-\Delta)^{\alpha / 2} u(t)\right]^{2} d x+\int_{\Omega} F(u(t)) d x-\int_{\Omega} g(x) u(t) d x
$$

is non-increasing in time. Note also that, thanks to the assumption (1.5),

$$
\forall \lambda_{1}>\delta>0 \quad \exists C_{\delta}>0 \quad \forall s \in \mathbb{R} \quad-F(s) \leq \frac{1}{2}\left(\lambda_{1}-\delta\right) s^{2}+C_{\delta} .
$$

This last condition follows from the calculation (compare [21, p. 76])

$$
-F(s)-\frac{1}{2}\left(\lambda_{1}-\delta\right) s^{2}=\int_{0}^{s}\left[\frac{-f(\tau)}{\tau}-\left(\lambda_{1}-\delta\right)\right] \tau d \tau \leq C_{\delta},
$$

valid for positive $s$, and a symmetric estimate for negative $s$. It follows from the property $\mathcal{L}(u(t)) \leq \mathcal{L}\left(u_{0}\right), t \geq 0,(2.9)$ and (2.11) that

$$
\int_{\Omega}\left[(-\Delta)^{\alpha / 2} u(t)\right]^{2} d x \leq \operatorname{const}\left(\mathcal{L}\left(u_{0}\right)\right), \quad t \geq 0 .
$$

Thus, the $H_{2,\{D\}}^{\alpha}(\Omega)$ norm of the solution is bounded for $t \geq 0$, consequently the solution is global in time. Moreover, the Lyapunov function $\mathcal{L}$ is bounded from 
below and satisfies the following condition: $\mathcal{L}(u) \rightarrow \infty$ as $\|u\|_{H_{2,\{D\}}^{\alpha}(\Omega)}^{2} \rightarrow \infty$. Indeed

$$
\begin{array}{rl}
\mathcal{L}(u) \geq \frac{\delta}{8 \lambda_{1}} \int_{\Omega}\left[(-\Delta)^{\alpha / 2} u\right]^{2} d x+\frac{\delta}{8} \int_{\Omega} u^{2} & d x-C_{\delta}|\Omega|-c_{\delta} \int_{\Omega} g^{2} d x \\
& \geq \frac{\delta}{8 \lambda_{1}}\|u\|_{H_{2,\{D\}}^{\alpha}(\Omega)}^{2}+C\left(\|g\|_{L^{2}(\Omega)}^{2}\right) .
\end{array}
$$

2.4. Comparison of eigenvalues. We will discuss next the consequences of the condition (1.5). Due to the Poincaré inequality:

$$
\gamma_{1} \int_{\Omega} \phi^{2} d x \leq \int_{\Omega}\left[(-\Delta)^{1 / 2} \phi\right]^{2} d x, \quad \text { for all } \phi \in H_{0}^{1}(\Omega),
$$

and due to the 'moments inequality' (see e.g. [22, p. 28]), for $0 \leq \alpha \leq 1$, since the realization of $(-\Delta)^{\alpha}$ with Dirichlet boundary condition is self-adjoint and positive definite,

$$
\int_{\Omega}\left[(-\Delta)^{\alpha / 2} \phi\right]^{2} d x \leq\left(\int_{\Omega}\left[(-\Delta)^{1 / 2} \phi\right]^{2} d x\right)^{\alpha}\left(\int_{\Omega} \phi^{2} d x\right)^{1-\alpha} .
$$

Dividing (2.10) by $\int_{\Omega} \phi^{2} d x$ (non-zero element) and using the variational characterization of the first eigenvalues $\lambda_{1}, \gamma_{1}$ ( $\gamma_{1}$ corresponds to $-\Delta$ with Dirichlet boundary condition), we get

$$
\begin{array}{r}
\lambda_{1}=\inf _{\phi \in H_{2,\{D\}}^{\alpha}(\Omega)} \frac{\int_{\Omega}\left[(-\Delta)^{\alpha / 2} \phi\right]^{2} d x}{\int_{\Omega} \phi^{2} d x} \leq \inf _{\phi \in H_{0}^{1}(\Omega)} \frac{\left(\int_{\Omega}\left[(-\Delta)^{1 / 2} \phi\right]^{2} d x\right)^{\alpha}}{\left(\int_{\Omega} \phi^{2} d x\right)^{\alpha}} \\
=\left(\inf _{\phi \in H_{0}^{1}(\Omega)} \frac{\int_{\Omega}\left[(-\Delta)^{1 / 2} \phi\right]^{2} d x}{\int_{\Omega} \phi^{2} d x}\right)^{\alpha}=\gamma_{1}^{\alpha},
\end{array}
$$

since $H_{0}^{1}(\Omega)$ is dense in $H_{2,\{D\}}^{\alpha}(\Omega)$. This shows that $\gamma_{1}^{\alpha}$ is greater or equal than the first eigenvalue $\lambda_{1}$ of $(-\Delta)^{\alpha}$ with Dirichlet boundary condition.

2.5. Stationary solutions of (1.4). Assumption (1.5) gives us also simple estimate, in $H_{2,\{D\}}^{\alpha}(\Omega)$, of the stationary solutions of (1.4). Indeed, multiplying the corresponding to (1.4) stationary equation

$$
\left\{\begin{array}{l}
(-\Delta)^{\alpha} v+f(v)=g(x), \quad x \in \Omega, \\
\left.v\right|_{\partial \Omega}=0
\end{array}\right.
$$

by $v$ we get

$$
\int_{\Omega}\left[(-\Delta)^{\alpha / 2} v\right]^{2} d x+\left(-\lambda_{1}+\delta\right) \int_{\Omega} v^{2} d x \leq C_{\delta} \int_{\Omega} g^{2}(x) d x+C|\Omega|
$$


for some constants $C_{\delta}, C$; and using the estimate (2.11) we finally have

$$
\frac{\delta}{\lambda_{1}} \int_{\Omega}\left[(-\Delta)^{\alpha / 2} v\right]^{2} d x \leq C_{\delta} \int_{\Omega} g^{2}(x) d x+C|\Omega| .
$$

\section{Useful facts and inequalities}

In case of the Dirichlet problem in bounded domain (1.4), for the corresponding abstract operator, we are using probably the most common definition of the fractional power of operator that comes from [25]. Let $X$ be a Banach space and $A$ be a closed linear operator such that the resolvent set contains $(-\infty, 0)$ and the resolvent satisfies

$$
\exists M>0 \quad \forall \lambda>0 \quad\left\|\lambda(\lambda+A)^{-1}\right\| \leq M .
$$

When $0<\operatorname{Re} \alpha<1$, following Balakrishnan ([25, p. 299]), for $x \in D(A)$ we define

$$
A^{\alpha} v=\frac{\sin (\pi \alpha)}{\pi} \int_{0}^{\infty} s^{\alpha-1} A(s I+A)^{-1} v d s .
$$

This integral converges absolutely for $0<\operatorname{Re} \alpha<1$, represents a continuous operator from $D_{1}^{1}$ into $X$ and is analytic in $\alpha$ for $0<\operatorname{Re} \alpha<1$ ([26, p. 92]). Similar approach (but for positive operators) can be found in [32, Section 1.15] where nice description of that notion is given.

We next quote an estimate that is borrowed from [13]. It is a consequence of the Kato-Beurling-Deny inequality (see e.g. [16]). A version of the famous Kato-Beurling-Deny inequality will be proved below for completeness of the presentation. We shall focus here on $A=-\Delta_{D}$ on $L^{2}(\Omega), \Omega \subset \mathbb{R}^{n}$ being a bounded $C^{2}$ domain, which is a special case of the general theory in [16].

It is well known that $(s I+A)^{-1}, s \geq 0$, has a positive symmetric kernel $K_{s}=K_{s}(x, y), x, y \in \Omega$, satisfying the estimate in [31, 5.168, p. 210]. Also, the integral formula for fractional powers of $A$ (see $[32, \S 1.15 .1(6)]$ ) is given by (3.1).

Writing below for simplicity of the notation $\langle\cdot, \cdot\rangle_{L^{2}(\Omega)}$ for the $L^{2}(\Omega)$ product, we obtain

$$
\begin{aligned}
\left\langle A^{\alpha} v, v^{q-1}\right\rangle_{L^{2}(\Omega)} & =\left\langle\frac{\sin \pi \alpha}{\pi} \int_{0}^{+\infty} s^{\alpha-1}(s I+A-s I)(s I+A)^{-1} v d s, v^{q-1}\right\rangle_{L^{2}(\Omega)} \\
& =\frac{\sin \pi \alpha}{\pi} \int_{0}^{+\infty} s^{\alpha-1}\left(\left\|v^{q}\right\|_{L^{1}(\Omega)}-\left\langle s(s I+A)^{-1} v, v^{q-1}\right\rangle_{L^{2}(\Omega)}\right) d s
\end{aligned}
$$

for $v \in \mathcal{C}_{0}^{+}$, where $\mathcal{C}_{0}^{+}=\left\{\phi \in C^{2}(\bar{\Omega}): \phi \geq 0,\left.\phi\right|_{\partial \Omega}=0\right\}$. The properties of $K_{s}$ and an elementary inequality of $[16$, p. 68$]$

$$
(s-t)\left(s^{q-1}-t^{q-1}\right) \geq \frac{4(q-1)}{q^{2}}\left|s^{q / 2}-t^{q / 2}\right|^{2}, \quad s \geq 0, t \geq 0, q \geq 2,
$$


ensure next that

$$
\begin{aligned}
\left\|v^{q}\right\|_{L^{1}(\Omega)} & -\left\langle s(s I+A)^{-1} v, v^{q-1}\right\rangle_{L^{2}(\Omega)} \\
= & \left\|v^{q}\right\|_{L^{1}(\Omega)}-s \int_{\Omega \times \Omega} v^{q-1}(x) v(y) K_{s}(x, y) d x d y \\
= & \frac{s}{2} \int_{\Omega \times \Omega}[v(x)-v(y)]\left[v^{q-1}(x)-v^{q-1}(y)\right] K_{s}(x, y) d x d y \\
& +\left\|v^{q}\right\|_{L^{1}(\Omega)}-s \int_{\Omega \times \Omega} v^{q}(x) K_{s}(x, y) d x d y \\
= & \frac{s}{2} \int_{\Omega \times \Omega}[v(x)-v(y)]\left[v^{q-1}(x)-v^{q-1}(y)\right] K_{s}(x, y) d x d y \\
& +\left\|v^{q}\right\|_{L^{1}(\Omega)}-s\left\|(s I+A)^{-1}\left(v^{q}\right)\right\|_{L^{1}(\Omega)} \\
\geq & \frac{4(q-1)}{q^{2}}\left(\frac{s}{2} \int_{\Omega \times \Omega}\left|v^{q / 2}(x)-v^{q / 2}(y)\right|^{2} K_{s}(x, y) d x d y\right. \\
& \left.+\left\|v^{q}\right\|_{L^{1}(\Omega)}-s\left\|(s I+A)^{-1}\left(v^{q}\right)\right\|_{L^{1}(\Omega)}\right)
\end{aligned}
$$

for $s>0, q \geq 2, v \in \mathcal{C}_{0}^{+}$, where in the last line above we have used additionally the inequality $1 \geq 4(q-1) / q^{2}, q \geq 2$, and the contraction property of $A$ in $L^{1}(\Omega)$ (see [16, Theorem 1.3.5]), which guarantees that

$$
\left\|v^{q}\right\|_{L^{1}(\Omega)}-s\left\|(s I+A)^{-1}\left(v^{q}\right)\right\|_{L^{1}(\Omega)} \geq 0, \quad s>0, v \in \mathcal{C}_{0}^{+} .
$$

Similar calculations show that

$$
\begin{aligned}
\left\langle A^{\alpha}\left(v^{q / 2}\right), v^{q / 2}\right\rangle_{L^{2}(\Omega)} & \left\langle\frac{\sin \pi \alpha}{\pi} \int_{0}^{+\infty} s^{\alpha-1}(s I+A-s I)(s I+A)^{-1}\left(v^{q / 2}\right) d s, v^{q / 2}\right\rangle_{L^{2}(\Omega)} \\
= & \frac{\sin \pi \alpha}{\pi} \int_{0}^{+\infty} s^{\alpha-1}\left(\left\|v^{q}\right\|_{L^{1}(\Omega)}-\left\langle s(s I+A)^{-1}\left(v^{q / 2}\right), v^{q / 2}\right\rangle_{L^{2}(\Omega)}\right) d s \\
= & \frac{\sin \pi \alpha}{\pi} \int_{0}^{+\infty} s^{\alpha-1}\left(\left\|v^{q}\right\|_{L^{1}(\Omega)}-s \int_{\Omega \times \Omega} v^{q / 2}(x) v^{q / 2}(y) K_{s}(x, y) d x d y\right) d s, \\
= & \frac{\sin \pi \alpha}{\pi} \int_{0}^{+\infty} s^{\alpha-1}\left(\left\|v^{q}\right\|_{L^{1}(\Omega)}+\frac{s}{2} \int_{\Omega \times \Omega}\left|v^{q / 2}(x)-v^{q / 2}(y)\right|^{2} K_{s}(x, y) d x d y\right. \\
& \left.-s \int_{\Omega \times \Omega} v^{q}(x) K_{s}(x, y) d x d y\right) d s \\
= & \frac{\sin \pi \alpha}{\pi} \int_{0}^{+\infty} s^{\alpha-1}\left(\frac{s}{2} \int_{\Omega \times \Omega}\left|v^{q / 2}(x)-v^{q / 2}(y)\right|^{2} K_{s}(x, y) d x d y\right. \\
& \left.+\left\|v^{q}\right\|_{L^{1}(\Omega)}-s\left\|(s I+A)^{-1}\left(v^{q}\right)\right\|_{L^{1}(\Omega)}\right) d s,
\end{aligned}
$$

for $s>0, q \geq 2, v \in \mathcal{C}_{0}^{+}$. As a consequence of the relation (3.2) and the obvious estimates when $\alpha=0$ or $\alpha=1$ we obtain the proposition below. 
Proposition 3.1. Let $\Omega \subset \mathbb{R}^{N}$ be a bounded domain, $\partial \Omega \in C^{2}$, and $A=$ $-\Delta_{D}$ on $L^{2}(\Omega)$. Then, for $\alpha \in[0,1], q \in[2,+\infty)$ and $\phi \in \mathcal{C}_{0}^{+}$the following inequality holds:

$$
\int_{\Omega} A^{\alpha} \phi \phi^{q-1} d x=\int_{\Omega} A^{\alpha / 2} \phi A^{\alpha / 2}\left(\phi^{q-1}\right) d x \geq \frac{4(q-1)}{q^{2}} \int_{\Omega}\left[A^{\alpha / 2}\left(\phi^{q / 2}\right)\right]^{2} d x .
$$

Extension of (3.3) to functions with arbitrary sign. It is well known that the resolvent $\left(\lambda I-\Delta_{D}\right)^{-1}, \lambda>0$, preserves positivity (see [16, Theorem 1.3.5]). This property extends directly to the resolvent of $\left(-\Delta_{D}\right)^{\alpha}, \alpha \in$ $(0,1)$, because of the formula ([25, p. 319]):

$$
\left(\lambda I+\left(-\Delta_{D}\right)^{\alpha}\right)^{-1}=\frac{\sin \pi \alpha}{\pi} \int_{0}^{+\infty} \frac{\tau^{\alpha}}{\lambda^{2}+2 \lambda \tau^{\alpha} \cos \pi \alpha+\tau^{2 \alpha}}\left(\tau I-\Delta_{D}\right)^{-1} d \tau,
$$

for $\lambda>0$, since the denominator above is positive. Next, for $\phi \in X_{L^{2}}^{\alpha}$ with $|\phi|^{q-1} \in X_{L^{2}}^{\alpha / 2}$, Theorem 1.3.2 of [16] gives us that $|\phi| \in X_{L^{2}}^{\alpha / 2}$ and

$$
\int_{\Omega}\left(-\Delta_{D}\right)^{\alpha} \phi \operatorname{sgn} \phi|\phi|^{q-1} d x \geq \int_{\Omega}(-\Delta)^{\alpha / 2}(|\phi|)\left(-\Delta_{D}\right)^{\alpha / 2}\left(|\phi|^{q-1}\right) d x .
$$

Together with (3.3), the last estimate justifies that

Corollary 3.2. For $\alpha \in[0,1], q \in[2,+\infty), \phi \in X_{L^{2}}^{\alpha}$ and $|\phi|^{q-1} \in X_{L^{2}}^{\alpha / 2}$, the following estimate holds:

$$
\int_{\Omega}\left(-\Delta_{D}\right)^{\alpha} \phi \operatorname{sgn} \phi|\phi|^{q-1} d x \geq \frac{4(q-1)}{q^{2}} \int_{\Omega}\left[\left(-\Delta_{D}\right)^{\alpha / 2}\left(|\phi|^{q / 2}\right)\right]^{2} d x .
$$

REMARK 3.3. A number of estimates, similar to above, but in the case of the whole $\mathbb{R}^{N}$, were reported in the review article [24], see also the source references there. In particular the, corresponding to (3.3), (3.4), estimates in $\mathbb{R}^{N}$ called Strook-Varopoulos inequality can be found in [24] together with their proofs. In fact, a more general form of the operator; Lévy operator, appears in these estimates.

3.1. The Moser-Alikakos technique in bounded domain. The lemma below will be used in the estimate of the $L^{\infty}(\Omega)$ norm of the solution in the second example. Recall that $X=H_{p,\{D\}}^{-\alpha}(\Omega), X^{1+\varepsilon}=H_{p,\{D\}}^{\alpha+2 \varepsilon}(\Omega)$ in this example. We need to have the embedding $X^{1+\varepsilon} \subset L^{\infty}(\Omega)$, which holds provided that $\alpha+2 \varepsilon>N / p$. As known from the theory of the second order parabolic equations, if we want the solution to vary in $L^{\infty}(\Omega)$ (e.g. to have the Maximum Principle), we eventually need to take large value of $p \geq 2$ (especially when the space dimension $N$ is large) to fulfill the condition $\alpha+2 \varepsilon>N / p$. 
Lemma 3.4. For $X^{1+\varepsilon}=H_{p,\{D\}}^{\alpha+2 \varepsilon}(\Omega)$ solutions to (1.4), $\alpha+2 \varepsilon>N / p$ and $g \in L^{\infty}(\Omega)$, the following implication holds:

$$
\left(\left\|u\left(t, u_{0}\right)\right\|_{L^{1}(\Omega)} \leq \mathrm{const}, t \geq 0\right) \Rightarrow\left(\left\|u\left(t, u_{0}\right)\right\|_{L^{\infty}(\Omega)} \leq \text { const }^{\prime}, t \geq 0\right) .
$$

Observe that the dissipative condition (1.5) implies

$$
\exists C<\lambda_{1} \quad \exists D>0 \quad \forall s \in R \quad-s f(s) \leq C s^{2}+D,
$$

which in order corresponds to $[12,(9.3 .5)]$. Moreover, by the estimate (3.4) with $q=2^{k}$, we have

$$
\frac{\left(2^{k}-1\right)}{2^{2 k-2}} \int_{\Omega}\left[\left(-\Delta_{D}\right)^{\alpha / 2}\left(|\phi|^{2^{k-1}}\right)\right]^{2} d x \leq \int_{\Omega}\left[\left(-\Delta_{D}\right)^{\alpha} \phi\right]|\phi|^{2^{k}-1} \operatorname{sgn} \phi d x .
$$

With the above conditions (3.6), (3.7), we are able to repeat the calculations of [12, Lemma 9.3.1] and get (3.5). The calculation goes as follows.

Multiplying (1.4) by $u^{2^{k}-1}, k=1,2, \ldots$, we get

$$
\begin{aligned}
\frac{1}{2^{k}} \frac{d}{d t} \int_{\Omega} u^{2^{k}} d x=-\int_{\Omega}(-\Delta)^{\alpha} u|u|^{2^{k}-1} & \operatorname{sgn}(u) d x \\
& -\int_{\Omega} f(u) u u^{2^{k}-2} d x+\int_{\Omega} g(x) u^{2^{k}-1} d x .
\end{aligned}
$$

Then, thanks to (3.6) and (3.7) and an elementary estimate $s^{2^{k}-2} \leq s^{2^{k}}+1$, we obtain (with an equivalent norm of $H_{2,\{D\}}^{\alpha}(\Omega)$, in case of bounded $\Omega$ and Dirichlet boundary condition)

$$
\begin{aligned}
\frac{d}{d t} \int_{\Omega} u^{2^{k}} d x \leq & -\frac{2^{k}-1}{2^{k-2}} \int_{\Omega}\left[(-\Delta)^{\alpha / 2}\left(|u|^{2^{k-1}}\right)\right]^{2} d x \\
& +2^{k} \int_{\Omega}\left(C u^{2}+D\right) u^{2^{k}-2} d x+2^{k} \int_{\Omega} g(x) u^{2^{k}-1} d x \\
\leq & -\frac{2^{k}-1}{2^{k-2}}\left\||u|^{2^{k-1}}\right\|_{H_{2,\{D\}}^{2}(\Omega)}^{2} \\
& +2^{k}(C+D) \int_{\Omega} u^{2^{k}} d x+2^{k} D|\Omega|+2^{k} \int_{\Omega} g(x) u^{2^{k}-1} d x .
\end{aligned}
$$

Moreover, since

$$
\|\phi\|_{L^{2}(\Omega)} \leq\|\phi\|_{L^{2+\mu}(\Omega)}^{(2+\mu) /(2+2 \mu)}\|\phi\|_{L^{1}(\Omega)}^{\mu /(2+2 \mu)}, \quad \phi \in L^{1}(\Omega) \cap L^{2+\mu}(\Omega),
$$

and, for $0<\mu \leq 4 \alpha /(N-2 \alpha)$,

$$
\|\phi\|_{L^{2+\mu}(\Omega)} \leq c_{\mu}\|\phi\|_{H_{2}^{\alpha}(\Omega)}, \quad \phi \in H_{2}^{\alpha}(\Omega),
$$

the Young inequality lead to the estimate

$$
\begin{gathered}
\forall \delta>0 \quad \forall 0<\mu \leq 4 \alpha /(N-2 \alpha) \quad \exists C_{\delta, \mu}>0 \\
\|\phi\|_{L^{2}(\Omega)}^{2} \leq \delta\|\phi\|_{H_{2}^{\alpha}(\Omega)}^{2}+C_{\delta, \mu}\|\phi\|_{L^{1}(\Omega)}^{2}
\end{gathered}
$$


From (3.9) and (3.10) and Hölder inequality $\left(p=2^{k} /\left(2^{k}-1\right), q=2^{k}\right)$, we obtain

$$
\begin{aligned}
\frac{d}{d t} \int_{\Omega} u^{2^{k}} d x \leq & -\frac{2^{k}-1}{2^{k-2} \delta} \int_{\Omega} u^{2^{k}} d x+\frac{\left(2^{k}-1\right) C_{\delta, \mu}}{2^{k-2} \delta}\left(\int_{\Omega}|u|^{2^{k-1}} d x\right)^{2} \\
& +2^{k}(C+D) \int_{\Omega} u^{2^{k}} d x+2^{k} D|\Omega| \\
& +2^{k}\left(\int_{\Omega}|g(x)|^{2^{k}} d x\right)^{1 / 2^{k}}\left(\int_{\Omega} u^{2^{k}} d x\right)^{\left(2^{k}-1\right) / 2^{k}} \\
\leq & \left.\frac{-2}{\delta}+2^{k}(C+D)\right) \int_{\Omega} u^{2^{k}} d x+\frac{4 C_{\delta, \mu}}{\delta}\left(\int_{\Omega}|u|^{2^{k-1}} d x\right)^{2} \\
& +2^{k} D|\Omega|+2^{k}\left(C_{\varepsilon} \int_{\Omega}|g(x)|^{2^{k}} d x+\varepsilon \int_{\Omega} u^{2^{k}} d x\right),
\end{aligned}
$$

the last by the Young inequality. Setting $\delta=2^{1-k} /(C+D+2), \varepsilon=1$, we obtain

$$
\begin{aligned}
\frac{d}{d t} \int_{\Omega} u^{2^{k}} d x \leq-2^{k} \int_{\Omega} u^{2^{k}} d x+2^{k+1} \bar{C}_{\delta, \mu} & \left(\int_{\Omega}|u|^{2^{k-1}} d x\right)^{2} \\
& +2^{k} D|\Omega|+2^{k} C_{\varepsilon} \int_{\Omega}|g(x)|^{2^{k}} d x
\end{aligned}
$$

which leads, just as in $[12,(9.3 .13)]$, to the final estimate

$$
\sup _{t \geq 0}\|u(t)\|_{L^{\infty}(\Omega)} \leq \text { const } \max \left\{\sup _{t \geq 0}\|u(t)\|_{L^{1}(\Omega)}, 1\right\} .
$$

REMARK 3.5. A version of Lemma 3.4 stays valid if instead of the $L^{1}(\Omega)$ estimate we know a $L^{2^{r}}(\Omega)$ (with fixed $r \in \mathbb{N}$ ) estimate of $u\left(t, u_{0}\right)$. The induction argument will start from the $L^{2^{r}}(\Omega)$ norm of the solution in that case.

3.2. Moser-Alikakos technique in $\mathbb{R}^{N}$. If instead of a bounded domain $\Omega$ and homogeneous Dirichlet boundary condition we consider the Cauchy problem (1.1) in the whole of $\mathbb{R}^{N}$, condition (3.6) need to be replaced (from (2.2)) with

$$
\exists C<\lambda \quad \forall D>0 \quad \forall s \in \mathbb{R} \quad-s f(s) \leq C s^{2}+D|s|,
$$

and we need to have $g \in L^{1}\left(\mathbb{R}^{N}\right) \cap L^{\infty}\left(\mathbb{R}^{N}\right)$. We are also using another definition of the fractional powers of $(-\Delta)$ in that case, following [18], [15], [24]:

Definition 3.6. For every $\beta \in(0,1)$ we set:

$$
(-\Delta)^{\beta} v(x)=-C(\beta) \lim _{\varepsilon \rightarrow 0} \int_{|z| \geq \varepsilon} \frac{v(x-z)-v(x)}{|z|^{N+2 \beta}} d z,
$$

valid for $v \in \mathcal{S}$, the Schwartz class. An extension to functions $v \in C_{B}^{2}\left(\mathbb{R}^{N}\right)$ is discussed in [18]. 
We thus consider the problem (1.1)

$$
\begin{cases}u_{t}+(-\Delta)^{\alpha} u+f(u)+\lambda u=g(x), & t>0, x \in \mathbb{R}^{N}, \\ u(0, x)=u_{0}(x), & x \in \mathbb{R}^{N},\end{cases}
$$

under the assumption (3.11), and with $g \in L^{1}\left(\mathbb{R}^{N}\right) \cap L^{\infty}\left(\mathbb{R}^{N}\right)$. Unfortunately the $H^{\alpha}\left(\mathbb{R}^{N}\right) \cap L^{p+1}\left(\mathbb{R}^{N}\right)$ solution to (1.1) constructed above is too weak to vary in $L^{\infty}\left(\mathbb{R}^{N}\right)$, and to proceed with the estimates below we need to assure that the solution vary in $L^{p+1}\left(\mathbb{R}^{N}\right) \cap L^{\infty}\left(\mathbb{R}^{N}\right)$. But one can work with the $H_{p+1}^{\beta}\left(\mathbb{R}^{N}\right)$ solution of $(1.1)$ with $\beta>N /(p+1)$ to have enough smoothness for such calculations. Note also, that the assumption (3.11) is weaker than the left hand side of the condition (1.2), so that assuming (1.2) we can use a weaker condition (3.11) instead.

Multiplying (1.1) by $u^{2 l-1} ; 2 l \geq p+2, l \in \mathbb{N}$, we obtain:

$$
\begin{aligned}
\int_{\mathbb{R}^{N}} u_{t} u^{2 l-1} d x+ & \int_{\mathbb{R}^{N}}(-\Delta)^{\alpha} u u^{2 l-1} d x \\
& +\int_{\mathbb{R}^{N}} f(u) u u^{2 l-2} d x+\lambda \int_{\mathbb{R}^{N}} u^{2 l} d x=\int_{\mathbb{R}^{N}} g(x) u^{2 l-1} d x .
\end{aligned}
$$

The second term is non-negative thanks to Corollary 3.2. Recall that we are working with the solutions of (1.1) varying in $L^{p+1}\left(\mathbb{R}^{N}\right) \cap L^{\infty}\left(\mathbb{R}^{N}\right)$. Also, the nonlinear term is estimated with the use of an elementary inequality

$$
\forall s \in \mathbb{R} \quad \forall \delta>0 \quad|s|^{2 l-1} \leq \delta^{-1} s^{2 l}+\delta^{2 l-2-p}|s|^{p+1}
$$

and (3.11) as follows:

$$
-\int_{\mathbb{R}^{N}} f(u) u u^{2 l-2} d x \leq \int_{\mathbb{R}^{N}}\left(C+D \delta^{-1}\right) u^{2 l} d x+D \delta^{2 l-2-p} \int_{\mathbb{R}^{N}}|u|^{p+1} d x,
$$

and the right hand side term is estimated with the use of the Hölder and Young inequality. We thus obtain

$$
\begin{aligned}
\frac{1}{2 l} \frac{d}{d t} \int_{\mathbb{R}^{N}} u^{2 l} d x \leq\left(C+D \delta^{-1}\right. & +\varepsilon-\lambda) \int_{\mathbb{R}^{N}} u^{2 l} d x \\
& +D \delta^{2 l-2-p} \int_{\mathbb{R}^{N}}|u|^{p+1} d x+C_{\varepsilon} \int_{\mathbb{R}^{N}}|g(x)|^{2 l} d x .
\end{aligned}
$$

Since $C<\lambda$, we can choose $\delta=\delta_{0}=4 D /(\lambda-C)$ and $\varepsilon=\varepsilon_{0}=(\lambda-C) / 4$ (here $C_{\varepsilon}=$ const $\varepsilon^{-2 l}$ comes from the Young inequality) to have $C+D \delta^{-1}+\varepsilon-\lambda=$ $(C-\lambda) / 2<0$. Solving the differential inequality we obtain:

$$
\begin{aligned}
& \int_{\mathbb{R}^{N}} u^{2 l} d x \leq\left[\int_{\mathbb{R}^{N}} u_{0}^{2 l} d x\right. \\
+ & \left.\int_{0}^{t} e^{-l(C-\lambda) s}\left(2 l D \delta_{0}^{2 l-2-p} \int_{\mathbb{R}^{N}}|u(s)|^{p+1} d x+2 l C_{\varepsilon_{0}}\|g\|_{L^{2 l}\left(\mathbb{R}^{N}\right)}^{2 l}\right) d s\right] e^{l(C-\lambda) t} .
\end{aligned}
$$


If $\sup _{s \in[0, T]}\|u(s)\|_{L^{p+1}\left(\mathbb{R}^{N}\right)} \leq M$, then taking the $2 l$ roots we find that:

$$
\begin{aligned}
\| u(t) & \left\|_{L^{2 l}\left(\mathbb{R}^{N}\right)} \leq\right\| u_{0} \|_{L^{2 l}\left(\mathbb{R}^{N}\right)} e^{(C-\lambda) t / 2} \\
& +\left((2 l D)^{-2 l} \delta_{0}^{1-(2+p) /(2 l)} M^{(p+1) /(2 l)}+\left(2 l C_{\varepsilon_{0}}\right)^{-2 l}\|g\|_{L^{2 l}\left(\mathbb{R}^{N}\right)}\right) \\
& \cdot\left(\int_{0}^{t} e^{l(C-\lambda)(t-s)} d s\right)^{1 /(2 l)} \\
\leq & \left\|u_{0}\right\|_{L^{2 l}\left(\mathbb{R}^{N}\right)} e^{(C-\lambda) t / 2} \\
& +\left((2 l D)^{-2 l} \delta_{0}^{1-(2+p) /(2 l)} M^{(p+1) /(2 l)}+\left(2 l C_{\varepsilon_{0}}\right)^{-2 l}\|g\|_{L^{2 l}\left(\mathbb{R}^{N}\right)}\right) \\
& \cdot\left(\frac{1-e^{l(C-\lambda) t}}{-l(C-\lambda)}\right)^{1 /(2 l)} .
\end{aligned}
$$

We can let $l \rightarrow+\infty$ in (3.12) to get the required $L^{\infty}\left(\mathbb{R}^{N}\right)$ bound:

$$
\|u(t)\|_{L^{\infty}\left(\mathbb{R}^{N}\right)} \leq\left\|u_{0}\right\|_{L^{\infty}\left(\mathbb{R}^{N}\right)} e^{(C-\lambda) t / 2}+\delta_{0}+\|g\|_{L^{\infty}\left(\mathbb{R}^{N}\right)},
$$

valid for $t \in[0, T]$.

3.3. $L^{p+1}\left(\mathbb{R}^{N}\right)$ a priori estimate. To complete the induction argument, we give below the $L^{p+1}\left(\mathbb{R}^{N}\right)$ a priori estimate of the solution $u$ of (1.1). Multiplying (1.1) by $|u|^{p} \operatorname{sgn}(u)$, we obtain

$$
\begin{aligned}
\int_{\mathbb{R}^{N}} u_{t}|u|^{p} \operatorname{sgn}(u) d x+\int_{\mathbb{R}^{N}}(-\Delta)^{\alpha} u|u|^{p-1} u d x+\int_{\mathbb{R}^{N}} f(u) u|u|^{p-1} d x \\
+\lambda \int_{\mathbb{R}^{N}}|u|^{p+1} d x=\int_{\mathbb{R}^{N}} g|u|^{p} \operatorname{sgn}(u) d x .
\end{aligned}
$$

Since $u,(-\Delta)^{\alpha} u \in L^{p+1}\left(\mathbb{R}^{N}\right)$ we infer from Lemma 4.9 that the second term is non-negative. Then, thanks to the Young inequality and the simplified assumption

$$
\exists 0 \leq C_{0}<\lambda \quad \forall s \in \mathbb{R} \quad-f(s) s \leq C_{0} s^{2},
$$

we get

$$
\frac{1}{p+1} \frac{d}{d t} \int_{\mathbb{R}^{N}}|u|^{p+1} d x+\left(\lambda-C_{0}-\delta\right) \int_{\mathbb{R}^{N}}|u|^{p+1} d x \leq C_{\delta} \int_{\mathbb{R}^{N}}|g|^{p+1} d x,
$$

where $\delta>0$ is sufficiently small, such that $M_{1}=\lambda-C_{0}-\delta$ is positive. Consequently,

$$
\begin{array}{r}
\|u(t)\|_{L^{p+1}\left(\mathbb{R}^{N}\right)}^{p+1} \leq\left(\|u(0)\|_{L^{p+1}\left(\mathbb{R}^{N}\right)}^{p+1}-\frac{C_{\delta}\|g\|_{L^{p+1}\left(\mathbb{R}^{N}\right)}^{p+1}}{M_{1}}\right) e^{-(p+1) M_{1} t} \\
+\frac{C_{\delta}\|g\|_{L^{p+1}\left(\mathbb{R}^{N}\right)}^{p+1}}{M_{1}} .
\end{array}
$$


3.4. $L^{1}\left(\mathbb{R}^{N}\right)$ a priori estimate. Under the simplified assumption (3.14) it is possible to estimate the $L^{1}\left(\mathbb{R}^{N}\right)$ norm of the solution to (1.1). Multiplying by $\operatorname{sgn}(u)$ and using the fractal generalization of the famous Kato inequality (see [24, Theorem 1.20]):

$$
\int_{\mathbb{R}^{N}}(-\Delta)^{\alpha} \phi \operatorname{sgn}(\phi) d x \geq 0, \quad \alpha \in(0,1], \quad \phi \in C_{0}^{\infty}\left(\mathbb{R}^{N}\right),
$$

we find that

$$
\frac{d}{d t} \int_{\mathbb{R}^{N}}|u| d x+\lambda \int_{\mathbb{R}^{N}}|u| d x \leq-\int_{\mathbb{R}^{N}} f(u) \operatorname{sgn}(u) d x+\int_{\mathbb{R}^{N}}|g| d x .
$$

When $g \in L^{1}\left(\mathbb{R}^{N}\right)$, it follows from (3.14) that $-f(u) \operatorname{sgn}(u) \leq C_{0}|u|$, and, we obtain the estimate

$$
\|u\|_{L^{1}\left(\mathbb{R}^{N}\right)} \leq\left\|u_{0}\right\|_{L^{1}\left(\mathbb{R}^{N}\right)} e^{-\left(\lambda-C_{0}\right) t}+\|g\|_{L^{1}\left(\mathbb{R}^{N}\right)} \frac{1-e^{-\left(\lambda-C_{0}\right) t}}{\lambda-C_{0}} .
$$

3.5. Some properties of the cut-off function. We consider the smooth (at least $C^{2}$, but we prefer $\theta \in C^{\infty}$ ) cut-off function $\theta: \mathbb{R}^{N} \rightarrow[0,1]$,

$$
\theta(x)= \begin{cases}1, & |x| \geq 2 \\ 0, & |x| \leq 1\end{cases}
$$

Next we discuss a property of the cut-off function which is important in further estimates.

Lemma 3.7. Let $\Lambda=(-\Delta)^{1 / 2}$, then for any $\alpha \in(0,1)$, there exists a constant $M=M(\alpha, N, \theta)>0$ such that

$$
\left|\Lambda^{2 \alpha} \theta(x)\right| \leq M<\infty \quad \text { for all } x \in \mathbb{R}^{N} .
$$

Proof. By $\left[18\right.$, p. 302], since $\theta \in C_{B}^{2}\left(\mathbb{R}^{N}\right)$, we have

$$
\begin{aligned}
& -\Lambda^{2 \alpha} \theta(x)=c_{N}(\alpha) \int_{|z|<1} \frac{\theta(x-z)-\theta(x)+\nabla \theta(x) \cdot z}{|z|^{N+2 \alpha}} d z \\
& \quad+c_{N}(\alpha) \int_{|z| \geq 1} \frac{\theta(x-z)-\theta(x)}{|z|^{N+2 \alpha}} d z=: I_{1}+I_{2},
\end{aligned}
$$

where $c_{N}(\alpha)=\alpha \Gamma(N / 2+\alpha) /\left(\pi^{N / 2+2 \alpha} \Gamma(1-\alpha)\right)$.

Note that $\theta(\cdot) \in[0,1]$, so that we have

$$
\left|I_{2}\right| \leq c_{N}(\alpha) \int_{|z| \geq 1} \frac{1}{|z|^{N+2 \alpha}} d z \leq \operatorname{const}(\alpha, N)<\infty
$$

for each $\alpha \in(0,1)$.

On the other hand, from the Taylor formula

$$
\theta(x-z)-\theta(x)+\nabla \theta(x) \cdot z=\frac{1}{2} \theta^{\prime \prime}(\xi) \cdot z^{2},
$$


where $\theta^{\prime \prime}(\xi)$ denote the Hessian matrix of $\theta$, we have (here we need to use the notion of the Cauchy principal value integral to justify the calculations),

$$
\begin{aligned}
I_{1} & =c_{N}(\alpha) P V \int_{|z|<1} \frac{\theta(x-z)-\theta(x)+\nabla \theta(x) \cdot z}{|z|^{N+2 \alpha}} d z \\
& =c_{N}(\alpha) \int_{|z|<1} \frac{\theta^{\prime \prime}(\xi) \cdot z^{2} / 2}{|z|^{N+2 \alpha}} d z
\end{aligned}
$$

and so

$$
\begin{aligned}
\left|I_{1}\right| & \leq c_{N}(\alpha) \int_{|z|<1} \frac{\left|\theta^{\prime \prime}(\xi) \cdot z^{2}\right| / 2}{|z|^{N+2 \alpha}} d z \\
& \leq c(N, \alpha, \theta) \int_{|z|<1} \frac{|z|^{2}}{|z|^{N+2 \alpha}} d z \leq C(N, \theta, \alpha)<\infty .
\end{aligned}
$$

Let $\theta$ be as above, moreover, we set $\theta_{k}(\cdot)=\theta(\cdot / k), k=1,2, \ldots$ Then the following identity is obvious (e.g. see [18]):

Lemma 3.8. For any $s \in(0,2)$,

$$
\Lambda^{s} \theta_{k}(x)=\left.\frac{1}{k^{s}} \Lambda^{s} \theta(z)\right|_{z=x / k}
$$

We will need also the following property of the operator $\Lambda^{2 \alpha}$ :

LEMMA 3.9. The operator $\Lambda^{2 \alpha}$ is symmetric in $L^{2}\left(\mathbb{R}^{N}\right)$.

Proof. When $f, g \in D\left(\Lambda^{2 \alpha}\right)=H^{2 \alpha}\left(\mathbb{R}^{N}\right)$, then we have

$$
\begin{aligned}
& \int_{\mathbb{R}^{N}} \Lambda^{2 \alpha} f(x) \cdot g(x) d x=-c_{N}(\alpha) \int_{\mathbb{R}^{N}} \int_{\mathbb{R}^{N}}\left[\frac{f(x-z) g(x)}{|z|^{N+2 \alpha}}-\frac{f(x) g(x)}{|z|^{N+2 \alpha}}\right] d z d x \\
& =-c_{N}(\alpha) \int_{\mathbb{R}^{N}} \frac{1}{|z|^{N+2 \alpha}}\left(\int_{\mathbb{R}^{N}} f(x) g(x-z) d x-\int_{\mathbb{R}^{N}} f(x) g(x) d x\right) d z \\
& =\int_{\mathbb{R}^{N}} f(x) \cdot \Lambda^{2 \alpha} g(x) d x
\end{aligned}
$$

which proves the claim.

\section{Attractor for the semigroup of solutions to (1.1)}

Now we discuss the dynamics of (1.1) in term of the global attractor. The following assumptions will be used throughout this section:

Assumption 4.1. Let $\alpha \in(0,1), g \in H^{-\alpha}\left(\mathbb{R}^{N}\right), f \in C^{1}(\mathbb{R})$ and satisfying (1.2)-(1.3). Moreover, we denote by $\{S(t)\}_{t \geq 0}$ the semigroup corresponding to the weak solution of (1.1) with initial data $u_{0} \in L^{2}\left(\mathbb{R}^{N}\right)$.

Then we know that

$$
S(\cdot):[0, \infty) \times L^{2}\left(\mathbb{R}^{N}\right) \ni\left(t, u_{0}\right) \mapsto u(t) \in L^{2}\left(\mathbb{R}^{N}\right)
$$

is continous. 
4.1. Dissipation of $\{S(t)\}_{t \geq 0}$ in $L^{2}\left(\mathbb{R}^{N}\right)$. Multiplying (1.1) by $u$ and integrating over $\mathbb{R}^{N}$ (here and after, we will calculate on strong solutions; the estimates will also be valid for the weak solutions by passing to the limits), we obtain that

$$
\frac{1}{2} \frac{d}{d t} \int_{\mathbb{R}^{N}}|u|^{2} d x+\int_{\mathbb{R}^{N}}\left(\Lambda^{\alpha} u\right)^{2} d x+\int_{\mathbb{R}^{N}} f(u) u d x+\lambda\|u\|^{2}=\int_{\mathbb{R}^{N}} g(x) u d x,
$$

where, for simplicity of further notation, $\|\cdot\|$ denotes the usual $L^{2}\left(\mathbb{R}^{N}\right)$-norm. Then, by the assumption (1.2), we have

$\frac{1}{2} \frac{d}{d t}\|u\|^{2}+\left\|\Lambda^{\alpha} u\right\|^{2}+c_{1} \int_{\mathbb{R}^{N}}|u|^{p+1} d x+\left(\lambda-k_{1}\right)\|u\|^{2} \leq\|g\|_{H^{-\alpha}\left(\mathbb{R}^{N}\right)}\|u\|_{H^{\alpha}\left(\mathbb{R}^{N}\right)}$.

Hence, noticing that $\|\cdot\|_{H^{\alpha}\left(\mathbb{R}^{N}\right)}^{2}=\left\|\Lambda^{\alpha} \cdot\right\|^{2}+\|\cdot\|^{2}$ and $\lambda>k_{1}$, using the Cauchy-Schwartz inequality, we can deduce that

$$
\frac{d}{d t}\|u\|^{2}+\left\|\Lambda^{\alpha} u\right\|^{2}+2 c_{1} \int_{\mathbb{R}^{N}}|u|^{p+1} d x+\left(\lambda-k_{1}\right)\|u\|^{2} \leq c_{\lambda, k_{1}}\|g\|_{H^{-\alpha}\left(\mathbb{R}^{N}\right)}^{2},
$$

which implies that

$$
\int_{0}^{t}\left(\|u(s)\|_{H^{\alpha}\left(\mathbb{R}^{N}\right)}^{2}+\|u(s)\|_{L^{p+1}\left(\mathbb{R}^{N}\right)}^{p+1}\right) d s \leq C_{\lambda, k_{1}, c_{1}}\left(t\|g\|_{H^{-\alpha}\left(\mathbb{R}^{N}\right)}^{2}+\left\|u_{0}\right\|^{2}\right)
$$

for any $t \geq 0$, and

$$
\|u(t)\|^{2} \leq e^{-\left(\lambda-k_{1}\right) t}\left\|u_{0}\right\|^{2}+\frac{c_{\lambda, k_{1}}\|g\|_{H^{-\alpha}\left(\mathbb{R}^{N}\right)}^{2}}{\lambda-k_{1}} \quad \text { for all } t \geq 0 .
$$

Obviously, the estimate (4.4) implies that

$$
B=\left\{u \in L^{2}\left(\mathbb{R}^{N}\right):\|u\|^{2} \leq \frac{c_{\lambda, k_{1}}\|g\|_{H^{-\alpha}\left(\mathbb{R}^{N}\right)}^{2}}{\lambda-k_{1}}+1\right\}
$$

is a bounded absorbing set for the semigroup $\{S(t)\}_{t \geq 0}$ in $L^{2}\left(\mathbb{R}^{N}\right)$. Then, by the standard theory of dynamical system (e.g. [12], [22]), we have the following result:

THEOREM 4.2 (Absorbing set). Under the Assumption 4.1, the semigroup $\{S(t)\}_{t \geq 0}$ has a bounded positively invariant absorbing set $B_{0}$ in $L^{2}\left(\mathbb{R}^{N}\right)$.

4.2. Asymptotic compactness in $L^{2}\left(\mathbb{R}^{N}\right)$. We will use the tail estimate technique (e.g. see [28], [33]) to get the asymptotic compactness.

TheOREM 4.3 (Tail estimate). In addition to the Assumption 4.1, let further $g \in L^{2}\left(\mathbb{R}^{N}\right)$. Then, for any $\varepsilon>0$, there exist positive constants $h=h\left(\varepsilon,\left\|B_{0}\right\|\right)$ and $T=T\left(\varepsilon,\left\|B_{0}\right\|\right)$ such that

$$
\int_{\mathcal{O}_{h}}\left|S(t) u_{0}\right|^{2} d x<\varepsilon \quad \text { for all } t \geq T \text { and } u_{0} \in B_{0},
$$


where $\mathcal{O}_{h}=\left\{x \in \mathbb{R}^{N}:|x| \geq h\right\}$ and $B_{0}$ is the positively invariant absorbing set obtained in Theorem 4.2 .

Proof. Taking the cut-off function $\theta$ as in (3.16), multiplying (1.1) by $\theta_{k} u \in$ $H^{\alpha}\left(\mathbb{R}^{N}\right) \subset L^{2}\left(\mathbb{R}^{N}\right)$ (compare Observation 4.6) and integrating over $\mathbb{R}^{N}$, we obtain that

$$
\begin{aligned}
\int_{\mathbb{R}^{N}} u_{t} u \theta_{k} d x+\left\langle(-\Delta)^{\alpha} u, u \theta_{k}\right\rangle_{H^{-\alpha}\left(\mathbb{R}^{N}\right), H^{\alpha}\left(\mathbb{R}^{N}\right)}+\lambda \int_{\mathbb{R}^{N}} u^{2} \theta_{k} d x \\
=-\int_{\mathbb{R}^{N}} f(u) u \theta_{k} d x+\int_{\mathbb{R}^{N}} g(x) u \theta_{k} d x .
\end{aligned}
$$

The components are estimated next as follows:

$$
\int_{\mathbb{R}^{N}} u_{t} u \theta_{k} d x=\frac{1}{2} \frac{d}{d t} \int_{\mathbb{R}^{N}} u^{2} \theta_{k} d x
$$

by the Young inequality $\left(\left|g \theta_{k}^{1 / 2} u \theta_{k}^{1 / 2}\right| \leq \delta u^{2} \theta_{k}+C_{\delta} g^{2} \theta_{k}, \delta>0\right)$, we have

$$
\left|\int_{\mathbb{R}^{N}} g(x) u \theta_{k} d x\right| \leq \delta \int_{\mathbb{R}^{N}} u^{2} \theta_{k} d x+C_{\delta} \int_{\mathbb{R}^{N}} g^{2} \theta_{k} d x
$$

for some small positive constant $\delta$; and from (1.2) we have

$$
-\int_{\mathbb{R}^{N}} f(u) u \theta_{k} d x \leq k_{1} \int_{\mathbb{R}^{N}} \theta_{k} u^{2} d x .
$$

We will need also the known pointwise estimate from [14], which states that:

$$
\forall 0 \leq \beta \leq 2 \quad \forall \phi \in C_{0}^{2}\left(\mathbb{R}^{N}\right) \quad 2 \phi \Lambda^{\beta} \phi(x) \geq \Lambda^{\beta} \phi^{2}(x) .
$$

Using (4.6) to the fractional term, due to the remark below, we obtain

$$
\left\langle(-\Delta)^{\alpha} u, u \theta_{k}\right\rangle_{H^{-\alpha}\left(\mathbb{R}^{N}\right), H^{\alpha}\left(\mathbb{R}^{N}\right)} \geq \frac{1}{2} \int_{\mathbb{R}^{N}} u^{2} \Lambda^{2 \alpha}\left(\theta_{k}\right) d x .
$$

At the same time, thanks to Lemmas 3.7 and 3.8, we have

$$
\left|\Lambda^{2 \alpha}\left(\theta_{k}\right)\right| \leq \frac{\text { const }}{k^{2 \alpha}}
$$

for some constant const which depends only on $N, \alpha$ and $\theta$.

Collecting the estimates (4.5)-(4.8) above, we thus get

$$
\frac{1}{2} \frac{d}{d t} \int_{\mathbb{R}^{N}} u^{2} \theta_{k} d x+\left(\lambda-k_{1}-\delta\right) \int_{\mathbb{R}^{N}} u^{2} \theta_{k} d x \leq \frac{\text { const }}{k^{2 \alpha}} \int_{\mathbb{R}^{N}} u^{2} d x+C_{\delta} \int_{\mathbb{R}^{N}} g^{2} \theta_{k} d x .
$$

Note that $\lambda>k_{1}$, so by taking $\delta \in\left(0,\left(\lambda-k_{1}\right) / 2\right)$ we have $\lambda-k_{1}-\delta \geq$ $\left(\lambda-k_{1}\right) / 2>0$ and

$$
\frac{d}{d t} \int_{\mathbb{R}^{N}} u^{2} \theta_{k} d x+\left(\lambda-k_{1}\right) \int_{\mathbb{R}^{N}} u^{2} \theta_{k} d x \leq \frac{\text { const } \cdot\left\|B_{0}\right\|^{2}}{k^{2 \alpha}}+C_{\delta} \int_{\mathbb{R}^{N}} g^{2} \theta_{k} d x
$$


for any $u_{0} \in B_{0}$. Therefore, for all $u_{0} \in B_{0}$ and all $t \geq 0$, we have

$$
\begin{aligned}
\int_{|x| \geq k} u^{2}(x, t) d x \leq & \int_{\mathbb{R}^{N}} u^{2}(x, t) \theta_{k} d x \\
& \leq e^{-\left(\lambda-k_{1}\right) t}\left\|B_{0}\right\|^{2}+\frac{\mathrm{const} \cdot\left\|B_{0}\right\|^{2}}{k^{2 \alpha}\left(\lambda-k_{1}\right)}+\frac{C_{\delta} \int_{\mathbb{R}^{N}} g^{2}(x) \theta_{k} d x}{\lambda-k_{1}}
\end{aligned}
$$

which allows us to complete the proof by noticing only that

$$
\int_{\mathbb{R}^{N}} g^{2} \theta_{k} d x \leq \int_{|x| \geq k} g^{2}(x) d x \rightarrow 0 \quad \text { as } k \rightarrow \infty .
$$

REMARK 4.4. The calculations in (4.7) will be justified using approximation argument. Since $u(t) \in H^{\alpha}\left(\mathbb{R}^{N}\right) \cap L^{p+1}\left(\mathbb{R}^{N}\right)$ (we fix $t>0$ here), there is a sequence $\left\{u_{n}\right\} \subset C_{0}^{\infty}\left(\mathbb{R}^{N}\right)$ convergent to $u(t)$ in $H^{\alpha}\left(\mathbb{R}^{N}\right)$. Note next that, since $u_{n} \in C_{0}^{\infty}\left(\mathbb{R}^{N}\right) \subset H^{2 \alpha}\left(\mathbb{R}^{N}\right)$, then $\Lambda^{2 \alpha} u_{n} \in L^{2}\left(\mathbb{R}^{N}\right)$. We are working with the triple of Hilbert spaces: $H^{\alpha}\left(\mathbb{R}^{N}\right) \subset L^{2}\left(\mathbb{R}^{N}\right) \subset H^{-\alpha}\left(\mathbb{R}^{N}\right)$, so that the linear functional (on $H^{\alpha}\left(\mathbb{R}^{N}\right)$ ) corresponding to $\Lambda^{2 \alpha} u_{n}$ is given by

$$
\left\langle\Lambda^{2 \alpha} u_{n}, \phi\right\rangle_{H^{-\alpha}\left(\mathbb{R}^{N}\right), H^{\alpha}\left(\mathbb{R}^{N}\right)}=\int_{\mathbb{R}^{N}} \Lambda^{2 \alpha} u_{n} \phi d x, \quad \phi \in H^{\alpha}\left(\mathbb{R}^{N}\right) .
$$

By (4.6) and the above observation $\left(u_{n} \theta_{k} \in H^{\alpha}\left(\mathbb{R}^{N}\right)\right.$ since $\theta_{k} \in C_{B}^{2}\left(\mathbb{R}^{N}\right)$; see Lemma 4.5)

(4.9) $\left\langle\Lambda^{2 \alpha} u_{n}, u_{n} \theta_{k}\right\rangle_{H^{-\alpha}\left(\mathbb{R}^{N}\right), H^{\alpha}\left(\mathbb{R}^{N}\right)}=\int_{\mathbb{R}^{N}} \Lambda^{2 \alpha} u_{n} u_{n} \theta_{k} d x \geq \frac{1}{2} \int_{\mathbb{R}^{N}} \Lambda^{2 \alpha}\left(u_{n}^{2}\right) \theta_{k} d x$.

Like in the proof of Lemma 3.9 we can show next that

$$
\frac{1}{2} \int_{\mathbb{R}^{N}} \Lambda^{2 \alpha}\left(u_{n}^{2}\right) \theta_{k} d x=\frac{1}{2} \int_{\mathbb{R}^{N}} u_{n}^{2} \Lambda^{2 \alpha}\left(\theta_{k}\right) d x .
$$

Finally, since $u(t) \in H^{\alpha}\left(\mathbb{R}^{N}\right)$, we can pass to the limits in the outline components (note that $u(t) \theta_{k} \in H^{\alpha}\left(\mathbb{R}^{N}\right)$ since $\theta_{k} \in C_{B}^{2}\left(\mathbb{R}^{N}\right)$ ), to obtain

$$
\left\langle\Lambda^{2 \alpha} u(t), u(t) \theta_{k}\right\rangle_{H^{-\alpha}\left(\mathbb{R}^{N}\right), H^{\alpha}\left(\mathbb{R}^{N}\right)} \geq \frac{1}{2} \int_{\mathbb{R}^{N}} u(t)^{2} \Lambda^{2 \alpha}\left(\theta_{k}\right) d x,
$$

which completes the calculations.

Passing to the limit in the left hand side of (4.9) we need the following lemma.

LEMma 4.5. Let $\left\{u_{n}\right\}_{n=1}^{\infty} \subset C_{0}^{\infty}\left(\mathbb{R}^{N}\right)$ and $u_{n} \rightarrow u$ in $H^{\alpha}\left(\mathbb{R}^{N}\right)$ as $n \rightarrow \infty$ $(\alpha \in(0,1))$. Then, for any (fixed) $\theta \in C_{B}^{2}\left(\mathbb{R}^{N}\right)$,

$$
\theta \cdot u_{n} \in H^{\alpha}\left(\mathbb{R}^{N}\right) \text { and } \theta \cdot u_{n} \rightarrow \theta \cdot u \quad \text { in } H^{\alpha}\left(\mathbb{R}^{N}\right) \text { as } n \rightarrow \infty .
$$

The above lemma follows immediately from the following more general observation: 
Observation 4.6. For any $0 \leq s \leq 1$ and $a \in C_{B}^{1}\left(\mathbb{R}^{N}\right)$ the multiplication $v \rightarrow$ av by $a$ is a bounded operator on $H^{s}\left(\mathbb{R}^{N}\right)$. Moreover,

$$
\exists C>0 \quad \forall v \in H^{s}\left(\mathbb{R}^{N}\right) \quad\|a v\|_{H^{s}\left(\mathbb{R}^{N}\right)} \leq C\|a\|_{C_{B}^{1}\left(\mathbb{R}^{N}\right)}^{s}\|a\|_{C_{B}^{0}\left(\mathbb{R}^{N}\right)}^{1-s}\|v\|_{H^{s}\left(\mathbb{R}^{N}\right)} .
$$

Proof. The following two estimates are evident:

$$
\begin{aligned}
\|a v\|_{L^{2}\left(\mathbb{R}^{N}\right)} & \leq\|a\|_{C_{B}^{0}\left(\mathbb{R}^{N}\right)}\|v\|_{L^{2}\left(\mathbb{R}^{N}\right)}, \\
\|a v\|_{H^{1}\left(\mathbb{R}^{N}\right)} & \leq\|a\|_{C_{B}^{1}\left(\mathbb{R}^{N}\right)}\|v\|_{H^{1}\left(\mathbb{R}^{N}\right)} .
\end{aligned}
$$

The rest is a consequence of the interpolation inequality.

Now, we are ready to prove the asymptotic compactness of $\{S(t)\}_{t \geq 0}$ in $L^{2}\left(\mathbb{R}^{N}\right)$.

Theorem 4.7 (Asymptotic compactness). Under the assumptions of Theorem 4.3 , the semigroup $\{S(t)\}_{t \geq 0}$ is asymptotically compact in $L^{2}\left(\mathbb{R}^{N}\right)$.

Proof. It is sufficient to show that for any $\varepsilon>0$, there is a $T=T(\varepsilon)>0$ such that

$$
S(t) B_{0} \text { has a finite } \varepsilon \text {-net in } L^{2}\left(\mathbb{R}^{N}\right), \quad \text { for all } t \geq T \text {; }
$$

recall that $B_{0}$ is the positively invariant absorbing set obtained in Theorem 4.2. By the invariance of $B_{0}$, we only need to prove that

$$
S(T) B_{0} \text { has a finite } \varepsilon \text {-net in } L^{2}\left(\mathbb{R}^{N}\right) \text { for some } T \text {. }
$$

For convenience, we divide our proof into steps.

Step 1. By Theorem 4.3 we know that there exist constants $T_{1}=T_{1}\left(\varepsilon,\left\|B_{0}\right\|\right)$ and $h_{1}=h_{1}\left(\varepsilon,\left\|B_{0}\right\|\right)$ such that

$$
\int_{|x| \geq h_{1}}\left|S(t) u_{0}\right|^{2} d x<\frac{\varepsilon}{4} \quad \text { for all } t \geq T_{1}, u_{0} \in B_{0} .
$$

Step 2. From (4.3) we have

$$
\begin{aligned}
& \int_{0}^{T_{1}}\left(\|u(s)\|_{H^{\alpha}\left(\mathbb{R}^{N}\right)}^{2}+\|u(s)\|_{L^{p+1}\left(\mathbb{R}^{N}\right)}^{p+1}\right) d s \\
& \quad \leq C_{\lambda, k_{1}, c_{1}}\left(\|g\|_{H^{-\alpha}\left(\mathbb{R}^{N}\right)}^{2} T_{1}+\left\|u_{0}\right\|^{2}\right),
\end{aligned}
$$

where $u_{0} \in B_{0}$ and $u(s)=S(s) u_{0}, s \in\left[0, T_{1}\right]$.

Set $S(\cdot) u_{0}$ for the function $S(\cdot) u_{0}: s \in\left[0, T_{1}\right] \rightarrow S(s) u_{0} \in L^{2}\left(\mathbb{R}^{N}\right)$, and denote

$$
B_{1}:=\left\{S(\cdot) u_{0}: u_{0} \in B_{0}\right\}
$$

Then (4.13) shows that

$$
B_{1} \text { is bounded in } L^{2}\left(0, T_{1} ; H^{\alpha}\left(\mathbb{R}^{N}\right)\right) \cap L^{p+1}\left(0, T_{1} ; L^{p+1}\left(\mathbb{R}^{N}\right)\right) .
$$


Consequently, due to the equation (1.1) (fulfilled in $H^{-\alpha}\left(\mathbb{R}^{N}\right)$ )

$$
u_{t}=-(-\Delta)^{\alpha} u-\lambda u-f(u)+g(x) \text {, }
$$

we also know that

(4.15) $\left\{u_{t}: u \in B_{1}\right\}$ is bounded in $L^{2}\left(0, T_{1} ; H^{-\alpha}\left(\mathbb{R}^{N}\right)\right)+L^{q}\left(0, T_{1} ; L^{q}\left(\mathbb{R}^{N}\right)\right)$,

where $1 / q+1 /(p+1)=1$.

Step 3. Let $u_{0 i} \in B_{0}$ and $u_{i}(t)=S(t) u_{0 i}, i=1,2$. Set $w(t)=u_{1}(t)-u_{2}(t)$, then we know that $w$ satisfies the following equation

$$
\left\{\begin{array}{l}
w_{t}+(-\Delta)^{\alpha} w+f\left(u_{1}\right)-f\left(u_{2}\right)+\lambda w=0, \\
w(0)=u_{01}-u_{02} .
\end{array}\right.
$$

Therefore, applying (1.3) and the inequality $\int_{\mathbb{R}^{N}}(-\Delta)^{\alpha} w w d x \geq 0$, we deduce that

$$
\left\|w\left(T_{1}\right)\right\|^{2} \leq e^{2(\lambda-l) T_{1}}\|w(s)\|^{2} \quad \text { for any } s \in\left[0, T_{1}\right]
$$

Step 4. Now we are ready to finish our proof by verifying (4.11) with $T=T_{1}$.

Note that (4.14), (4.15) imply that $\left.B_{1}\right|_{\left\{x \in \mathbb{R}^{N}:|x|<h_{1}\right\}}$ is bounded in $L^{2}\left(0, T_{1}\right.$; $\left.H^{\alpha}\left(\left\{x \in \mathbb{R}^{N}:|x|<h_{1}\right\}\right)\right) \cap L^{p+1}\left(0, T_{1} ; L^{p+1}\left(\left\{x \in \mathbb{R}^{N}:|x|<h_{1}\right\}\right)\right)\left(^{1}\right)$ and $\left.\left\{u_{t}: u \in B_{1}\right\}\right|_{\left\{x \in \mathbb{R}^{N}:|x|<h_{1}\right\}}$ is bounded in $L^{2}\left(0, T_{1} ; H^{-\alpha}\left(\left\{x \in \mathbb{R}^{N}:|x|<\right.\right.\right.$ $\left.\left.\left.h_{1}\right\}\right)\right)+L^{q}\left(0, T_{1} ; L^{q}\left(\left\{x \in \mathbb{R}^{N}:|x|<h_{1}\right\}\right)\right)$. Therefore (e.g. see [27]),

$$
\left.B_{1}\right|_{\left\{x \in \mathbb{R}^{N}:|x|<h_{1}\right\}} \text { is precompact in } L^{2}\left(0, T_{1} ; L^{2}\left(\left\{x \in \mathbb{R}^{N}:|x|<h_{1}\right\}\right) .\right.
$$

Hence, for such $\varepsilon>0$ and $T_{1}$, there exist $u_{i} \in B_{1}, i=1, \ldots, m_{\varepsilon}$, such that for any $u \in B_{1}$, there is some $u_{i}$ satisfying

$$
\int_{0}^{T_{1}} \int_{|x|<h_{1}}\left|u(x, s)-u_{i}(x, s)\right|^{2} d x d s \leq \frac{e^{-2 l T_{1}}}{2 T_{1}} \varepsilon,
$$

which, combining with (4.16) and (4.12), implies that

$$
\begin{aligned}
& \left\|u\left(T_{1}\right)-u_{i}\left(T_{1}\right)\right\|^{2}=\int_{\mathbb{R}^{N}}\left|u\left(x, T_{1}\right)-u_{i}\left(x, T_{1}\right)\right|^{2} d x \\
& =\int_{|x| \geq h_{1}}\left|u\left(x, T_{1}\right)-u_{i}\left(x, T_{1}\right)\right|^{2} d x+\int_{|x|<h_{1}}\left|u\left(x, T_{1}\right)-u_{i}\left(x, T_{1}\right)\right|^{2} d x \\
& \quad \leq 2 \int_{|x| \geq h_{1}}\left(\left|u\left(x, T_{1}\right)\right|^{2}+\left|u_{i}\left(x, T_{1}\right)\right|^{2}\right) d x+\frac{\varepsilon}{2}<2 \cdot \frac{\varepsilon}{4}+\frac{\varepsilon}{2}=\varepsilon,
\end{aligned}
$$

that is, $S\left(T_{1}\right) B_{0}$ has a finite $\varepsilon$-net in $L^{2}\left(\mathbb{R}^{N}\right)$.

$\left.{ }^{1}\right)$ The restriction of $H^{\alpha}\left(\mathbb{R}^{N}\right)$ to the space of functions defined in the ball is understand as in [32, Definition 4.2.1]. 
4.3. Global attractor in $L^{2}\left(\mathbb{R}^{N}\right)$. From Theorems 4.2 and 4.7 , and the continuity (4.1), applying the standard theory of dynamical systems, we can deduce our main result of this section immediately:

THEOREM 4.8. Under the assumptions of Theorem 4.7, $\{S(t)\}_{t \geq 0}$ has a global attractor $\mathcal{A}$ in $L^{2}\left(\mathbb{R}^{N}\right)$; that is, $\mathcal{A}$ is compact, invariant and attracts every $L^{2}\left(\mathbb{R}^{N}\right)$-bounded set in $L^{2}\left(\mathbb{R}^{N}\right)$-norm .

4.4. Further regularity for the attractor. In this subsection, similar to [30], applying the Moser-Alikakos technique (e.g. see [1]), we will deduce some higher regularity for the global attractor $\mathcal{A}$ through shifting it by a fixed point of the stationary equation.

Consider the stationary equation corresponding to (1.1):

$$
(-\Delta)^{\alpha} \phi+f(\phi)+\lambda \phi=g(x), \quad x \in \mathbb{R}^{N} .
$$

Then, from the assumptions (1.2)-(1.3) and $\lambda>k_{1}$, we know that (4.17) has at least one solution $\phi(x)$ which satisfies

$$
\phi \in H^{\alpha}\left(\mathbb{R}^{N}\right) \quad \text { and } \quad\|\phi\|_{H^{\alpha}\left(\mathbb{R}^{N}\right)} \leq M_{1}=M_{1}\left(\|g\|_{H^{-\alpha}\left(\mathbb{R}^{N}\right)}, \lambda-k_{1}\right)<\infty \text {. }
$$

Now, we decompose the solution $u(t)$ (obtained in Corollary 2.4) of (1.1) with initial data $u_{0} \in L^{2}\left(\mathbb{R}^{N}\right)$ as follows:

$$
u(t)=w(t)+\phi(x) \quad \text { for all } t \geq 0
$$

where $\phi$ is a fixed solution of (4.17); then $w(t)$ solves globally (a kind of weak solution as $u(t)$ for (1.1)) the following equation:

$$
\begin{cases}w_{t}+(-\Delta)^{\alpha} w+f(u)-f(\phi)+\lambda w=0, & (x, t) \in \mathbb{R}^{N} \times(0, \infty), \\ w(0, x)=u_{0}(x)-\phi(x), & x \in \mathbb{R}^{N}\end{cases}
$$

We first recall a lemma given in [23] (also [24, Remark 1.28], and Corollary 3.2 for bounded domains $\Omega$ ) for the fractional term:

Lemma 4.9 ([23]). Suppose that $\alpha \in(0,1), \beta \geq 2$ and $\varphi, \Lambda^{2 \alpha} \varphi \in L^{\beta}\left(\mathbb{R}^{N}\right)$. Then, the following inequality holds:

$$
\int_{\mathbb{R}^{N}}|\varphi|^{\beta-2} \varphi \Lambda^{2 \alpha} \varphi d x \geq \frac{2}{\beta} \int_{\mathbb{R}^{N}}\left(\Lambda^{\alpha}|\varphi|^{\beta / 2}\right)^{2} d x .
$$

Therefore, for each $\lambda>0$ and $\beta \geq 2$, we have the following equivalent norm of $|\varphi|^{\beta / 2}$ :

$$
\int_{\mathbb{R}^{N}}|\varphi|^{\beta-2} \varphi \Lambda^{2 \alpha} \varphi d x+\lambda \int_{\mathbb{R}^{N}}|\varphi|^{\beta} d x \geq \min \left\{\lambda, \frac{2}{\beta}\right\}\left\||\varphi|^{\beta / 2}\right\|_{H^{\alpha}\left(\mathbb{R}^{N}\right)}^{2} .
$$


Combining this with the embedding $H^{\alpha}\left(\mathbb{R}^{N}\right) \hookrightarrow L^{2 N /(N-2 \alpha)}\left(\mathbb{R}^{N}\right)(N \geq 2)$, we have

$$
\int_{\mathbb{R}^{N}}|\varphi|^{\beta-2} \varphi \Lambda^{2 \alpha} \varphi d x+\lambda \int_{\mathbb{R}^{N}}|\varphi|^{\beta} d x \geq \min \left\{\lambda, \frac{2}{\beta}\right\} \cdot C_{\alpha}\|\varphi\|_{L^{\beta N /(N-2 \alpha)}\left(\mathbb{R}^{N}\right)}^{\beta}
$$

where $C_{\alpha}$ is the embedding constant of $H^{\alpha}\left(\mathbb{R}^{N}\right) \hookrightarrow L^{2 N /(N-2 \alpha)}\left(\mathbb{R}^{N}\right)$.

Next, using the Moser-Alikakos technique, we prove by induction on $k(k=$ $0,1, \ldots)$ the existence of $T_{k}$, depending on $k$ and $\left\|B_{0}\right\|$, such that

$$
\int_{\mathbb{R}^{N}}|w(x, s)|^{2(N /(N-2 \alpha))^{k}} d x \leq R_{k} \quad \text { for any } u_{0} \in B_{0} \text { and } s \geq T_{k},
$$

and

$\left(B_{k}\right) \quad \int_{t}^{t+1} \int_{\mathbb{R}^{N}}|w(x, s)|^{2(N /(N-2 \alpha))^{k+1}} d x d s \leq R_{k}$

for any $u_{0} \in B_{0}$ and $t \geq T_{k}$,

where $R_{k}$ depends only on $k,\left\|B_{0}\right\|, C_{\alpha}, \lambda, l$ and $\|g\|_{H^{-\alpha}\left(\mathbb{R}^{N}\right)}$.

(i) Initialization of the induction $(k=0)$.

The estimate $\left(A_{0}\right)$ can be obtained from Theorem 4.2 and the estimate $\|w(t)\| \leq\|u(t)\|+\|\phi\|$; while $\left(B_{0}\right)$ can be proved exactly as that for (4.3): multiplying (4.18) by $w$, then using (1.3), $\left(A_{0}\right)$ and the embedding (4.19) with $\beta=2$.

(ii) The induction argument

We now assume the $\left(A_{k}\right)$ and $\left(B_{k}\right)$ hold for $k$, and we prove that the same is true for $k+1$.

Multiplying (4.18) by $|w|^{2(N /(N-2 \alpha))^{k+1}-2} \cdot w$ and integrating over $\mathbb{R}^{N}$, then we obtain that

$$
\begin{array}{r}
c_{k} \frac{d}{d t} \int_{\mathbb{R}^{N}}|w|^{2(N /(N-2 \alpha))^{k+1}} d x+\int_{\mathbb{R}^{N}}|w|^{2(N /(N-2 \alpha))^{k+1}-2} \cdot w \Lambda^{2 \alpha} w d x \\
+\lambda \int_{\mathbb{R}^{N}}|w|^{2(N /(N-2 \alpha))^{k+1}} d x \leq l \int_{\mathbb{R}^{N}}|w|^{2(N /(N-2 \alpha))^{k+1}} d x
\end{array}
$$

where the constant $c_{k}$ depends only on the spatial dimension $N$ and $k$, and we used (1.3).

Using $\left(B_{k}\right)$ and the Uniform Gronwall lemma, we infer from (4.20) that

$$
\int_{\mathbb{R}^{N}}|w(x, t)|^{2(N /(N-2 \alpha))^{k+1}} d x \leq R_{k+1}^{\prime} \quad \text { for any } t \geq T_{k}+1,
$$

which shows that $\left(A_{k+1}\right)$ is true. 
For $\left(B_{k+1}\right)$, we integrate (4.20) with respect to $t$ between $t$ and $t+1$, then we have

$$
\begin{aligned}
\int_{t}^{t+1} & \left(\int_{\mathbb{R}^{N}}|w|^{2(N /(N-2 \alpha))^{k+1}-2} \cdot w \Lambda^{2 \alpha} w d x\right. \\
& \left.+(\lambda-l) \int_{\mathbb{R}^{N}}|w|^{2(N /(N-2 \alpha))^{k+1}} d x\right) d s \leq R_{k+1}^{\prime \prime} \quad \text { for all } t \geq T_{k}+1
\end{aligned}
$$

where we have used (4.21). Hence, applying (4.19) with $\beta=2(N /(N-2 \alpha))^{k+1}$, we have

$$
c \int_{t}^{t+1}\left(\int_{\mathbb{R}^{N}}|w|^{2(N /(N-2 \alpha))^{k+2}} d x\right)^{(N-2 \alpha) / N} d s \leq R_{k+1}^{\prime \prime} \quad \text { for all } t \geq T_{k}+1
$$

and using the interpolation inequality for $L^{p}$ spaces we deduce $\left(B_{k+1}\right)$ immediately. Note that the exponent $k$ in $\left(A_{k}\right)$ can be arbitrary and $N /(N-2 \alpha)>1$, we indeed have proved the following asymptotic regularity result:

TheOREM 4.10. Under the Assumption 4.1, for any bounded $\left(\right.$ in $L^{2}\left(\mathbb{R}^{N}\right)$ ) subset $B \subset L^{2}\left(\mathbb{R}^{N}\right)$ and any $\delta \in[0, \infty)$, there exist constants $T_{\delta}=T(\|B\|, \delta)$ and $M_{\delta}<\infty$ such that

$$
\|S(t) B-\phi(x)\|_{L^{2+\delta}\left(\mathbb{R}^{N}\right)} \leq M_{\delta} \quad \text { for all } t \geq T_{\delta},
$$

where $\phi$ is a (fixed) solution of (4.17).

As a corollary of Theorem 4.10, we have the following integrability for the attractor $\mathcal{A}$ :

COROLlary 4.11. Under the assumptions of Theorem 4.8, then $\mathcal{A}-\phi(x)$ is bounded in $L^{2+\delta}\left(\mathbb{R}^{N}\right)$ for any $\delta \geq 0$.

Note that the $L^{2+\delta}\left(\mathbb{R}^{N}\right)$-bound of $\mathcal{A}-\phi(x)$ above will depends on $\delta$ since our forcing term $g$ belongs only to $L^{2}\left(\mathbb{R}^{N}\right)$.

4.5. Closing remarks. To avoid repetitions of the considerations concerning Cauchy's problem in $\mathbb{R}^{N}$, and to shorten the paper, we formulate without the proof a result concerning existence of the global attractor for (1.4). We only mention here that (1.4) fall into the class of the gradient systems (see [21], also [29], [10] for less restrictive definition).

Proposition 4.12. Let $u$ be an $\varepsilon$-regular solution to (1.4) and $p=2$. Assume that the conditions (1.5) and (1.6) are satisfied. Then the semigroup of global solutions generated by $(1.4)$ on $X^{1}=H_{2,\{D\}}^{\alpha}(\Omega)$ is dissipative in $H_{2,\{D\}}^{\alpha}(\Omega)$ and possesses a global attractor $\mathcal{A}$.

The proof of that property will follow the presentation in [10, pp. 712-713]. 
Acknowledgements. The paper was originated during C. Sun's visit at the Institute of Mathematics, Silesian University, Poland. We, the authors, are grateful to Professor G. Karch for sharing with us his knowledge on the recent developments concerning the subject of the paper. T.D. and M.B.K. were supported by NCN grant DEC-2012/05/B/ST1/00546 (Poland); C.S. was supported by the NSFC Grants 11031003, 11171028, lzujbky-2012-10 and NCET11-0214 (China).

\section{REFERENCES}

[1] A.D. Alikakos, An application of the invariance principle to reaction-diffusion equations, J. Differential Equations 33 (1979), 201-225.

[2] H. Amann, Linear and Quasilinear Parabolic Problems, Birkhäuser, Basel, 1995.

[3] J.M. Arrieta ANd A.N. Carvalho, Abstract parabolic problems with critical nonlinearities and applications to Navier-Stokes and heat equations, Trans. Amer. Math. Soc. 352 (1999), 285-310.

[4] C. Bardos, P. Penel, U. Frisch and P.-L. Sulem, Modified dissipativity for a nonlinear evolution equations arising in turbulence, Arch. Rational Mech. Anal. 71 (1979), $237-256$.

[5] P. Biler, G. Karch And W.A. Woyczynski, Asymptotics for multifractal conservation laws, Studia Math. 135 (1999), 231-252.

[6] H. Brezis, Operateurs Maximaux Monotones et Semi-groupes de Contractions dans les Espaces de Hilbert, North-Holland Publishing Company, Amsterdam, 1973.

[7] L. Cafarelli and L. Silvestre, An extension problem related to the fractional Laplacian, Comm. Partial Differential Equations 32 (2007), 1245-1260.

[8] A.N. Carvalho And J.W. Cholewa, Continuation and asymptotics of solutions to semilinear parabolic equations with critical nonlinearities, J. Math. Anal. Appl. 310 (2005), 557-578.

[9] A.N. Carvalho, J.W. Cholewa and T. Dlotko, Global attractors for problems with monotone operators, Boll. Unione Mat. Ital. (8) 2-B (1999), 693-706.

[10] A.N. Carvalho And T. Dlotko, Dynamics of the viscous Cahn-Hilliard equation, J. Math. Anal. Appl. 344 (2008), 703-725.

[11] A.N. Carvalho And C.B. Gentile, Comparison results for nonlinear parabolic equations with monotone principal part, J. Math. Anal. Appl. 259 (2001), 319-337.

[12] J.W. Cholewa And T. Dlotko, Global Attractors in Abstract Parabolic Problems, Cambridge University Press, Cambridge, 2000.

[13] J.W. Cholewa, T. Dlotko and A. Turski, Asymptotics of pseudodifferential parabolic equations, Demonstratio Math. 35 (2002), 75-91.

[14] A. Córdoba And D. Córdoba, A pointwise estimate for fractionary derivatives with applications to partial differential equations, Proc. Natl. Acad. Sci. USA 100 (2003), 15316-15317.

[15] P. Constantin, Euler Equations, Navier-Stokes Equations and Turbulence, Lecture Notes in Mathematics, vol. 1871, Springer-Verlag, Berlin, 2006, pp. 1-44.

[16] E.B. DAvies, Heat Kernels and Spectral Theory, Cambridge University Press, Cambridge, 1989.

[17] T. Dlotko And C. Sun, Dynamics of the modified viscous Cahn-Hilliard equation in $\mathbb{R}^{N}$, Topol. Methods Nonlinear. Anal. 35 (2010), 277-294. 
[18] J. Droniou and C. Imbert, Fractal first-order partial differential equations, Arch. Rational Mech. Anal. 182 (2006), 299-331.

[19] U. Frisch, M.F. Shlesinger and G.M. Zaslavsky (eds.), Lévy Flights and Related Topics in Physics, Springer-Verlag, Berlin, 1995.

[20] H. Gajewski, K. Gröger and K. Zacharias, Nichtlineare Operatorgleichungen und Operatordifferentialgleichungen, Akademie-Verlag, Berlin, 1974.

[21] J.K. Hale, Asymptotic Behavior of Dissipative Systems, Amer. Math. Soc., Providence, R.I., 1988.

[22] D. Henry, Geometric Theory of Semilinear Parabolic Equations, Springer-Verlag, Berlin, 1981.

[23] N. Ju, The maximum principle and the global attractor for the dissipative 2D quasigeostrophic equations, Commun. Math. Phys. 255 (2005), 161-181.

[24] G. KARCH, Nonlinear evolution equations with anomalous diffusion, Qualitative Properties of Solutions to Partial Differential Equations, J. Nečas Center for Mathematical Modeling, Charles University, Prague, 2009, pp. 25-65.

[25] H. Komatsu, Fractional powers of operators, Pacific J. Math. 19 (1966), 285-346.

[26] Fractional powers of operators II. Interpolation spaces, Pacific J. Math. 21 (1967), 89-111.

[27] J.L. Lions, Quelques Méthodes de Resolution des Problèmes aux Limites non Linéaires, Dunod, Paris, 1969.

[28] A. Rodriguez-Bernal and B. Wang, Attractors for partly dissipative reaction diffusion systems in $\mathbb{R}^{n}$, J. Math. Anal. Appl. 252 (2000), 790-803.

[29] G. RAugel, Global attractors in partial differential equations, Handbook of Dynamical Systems (B. Fiedler, ed.), vol. 2, Elsevier, Amsterdam, 2002, pp. 885-982.

[30] C. Sun, Asymptotic regularity for some dissipative equations, J. Differential Equations 248 (2010), 342-362.

[31] H. Tanabe, Functional Analytic Methods for Partial Differential Equations, Marcel Dekker, Inc., New York, 1997.

[32] H. Triebel, Interpolation Theory, Function Spaces, Differential Operators, Veb Deutscher Verlag, Berlin, 1978.

[33] B. WAng, Attractors for reaction-diffusion equations in unbounded domains, Physica D 128 (1999), 41-52.

[34] S. ZELIK, The attractor for a nonlinear reaction-diffusion system with a supercritical nonlinearity and its dimension, Rend. Accad. Naz. Sci. XL Mem. Mat. Appl. 24 (2000), $1-25$.

Tomasz Dlotko and Maria B. Kania

Institute of Mathematics

Silesian University

40-007 Katowice, POLAND

E-mail address: tdlotko@math.us.edu.pl, mkania@math.us.edu.pl

Chunyou Sun

School of Mathematics and Statistics

Lanzhou University

Lanzhou, 730000, P.R. CHINA

E-mail address: sunchy@lzu.edu.cn

TMNA : Volume $43-2014-\mathrm{N}^{\circ} 2$ 